Reprod. Nutr. Dévelop., 1987, 27 (1 B), 129-155.

\title{
L'ingestion alimentaire chez les ruminants : modalités et phénomènes associés
}

\author{
J.-P. DULPHY, Ph. FAVERDIN (*)
}

Laboratoire des Aliments,

I.N.R.A. Theix, 63122 Ceyrat, France.

(*) Station de Recherches sur la vache laitière,

I.N.R.A. Saint-Gilles, 35590 L'Hermitage, France.

Summary. Food intake in ruminants : modalities and associated phenomena.

This paper synthesizes the literature on modalities of food intake in ruminants and their main associated phenomena.

Firstly, circadian distribution of feeding and ruminating activities has been examined. Ruminants spend a large part of their time chewing. Their meals have been described in detail ; changes in rates of intake, time spent eating, the effect of restricting the amount fed or the period of feed accessibility have been discussed. When food is distributed, the animals have a "long " meal. These meals have been analysed in relation to the type of animal and the feed offered. The other meals (" small " meals) have been briefly described.

The paper next examines the phenomena associated with meals, or induced by them, and implied in the control of food intake. Forestomach motricity varies according to ruminant feeding behavior and plays a basic role in digesta transit. Rumen content varies with the meal and its chemical composition due to the arrival in the rumen of food, water and saliva. Rumination may require 600 to $650 \mathrm{~min} /$ day and is important in the comminution and sorting of rumen particles.

The digestive phenomena associated with meals are related to control of intake. The influence of rumen fill has been thoroughly discussed.

Finally, main humoral changes due to intake have been reviewed. The influence of volatile fatty acids (VFA) and metabolites has been discussed as well as the role of glucose, amino acids and fatty acids. Among the hormones, insulin and glucagon seem to play an important role in controlling food intake. The amounts of gastrointestinal hormones increase during intake and may also play an important part.

Despite a net improvement in the knowledge of phenomena related to intake, much still remains to be done in setting up models to describe these phenomena in relation to feeding activities and to aid in understanding the mechanisms controlling feed intake in ruminants.

L'étude de la quantité d'aliments ingérée, ainsi que celle de la répartition des repas, puis des modifications digestives et métaboliques qui les accompagnent, doit aider à la compréhension des mécanismes qui les contrôlent. II n'existe pas de synthèse récente sur ce sujet et, face à son ampleur, nous renvoyons le lecteur à des synthèses ponctuelles ou générales sur le comportement alimentaire (Gallouin et Focant, 1980) ou la régulation de l'ingestion chez le ruminant (Baile, 1979). 


\section{I. - Répartition des activités alimentaires et méryciques au cours du nycthémère.}

Les ruminants, comme la plupart des herbivores, passent de 3 à 12 h par jour à ingérer les quantités d'aliments nécessaires à leurs besoins.

Au pâturage, ils font deux grands repas, l'un après l'aube et l'autre avant le coucher du soleil (cf. Dulphy, Rémond et Thériez, 1979). Entre ces deux grands repas existent des repas diurnes intermédiaires. Une petite partie de l'ingestion a lieu la nuit, surtout si la journée a été cháude et en période de pleine lune (Ruckebusch et Buéno, 1978). Au milieu de la journée, les animaux se reposent et ruminent. En hiver les deux grands repas se chevauchent et la rumination a lieu principalement la nuit.

En stabulation, les animaux alimentés en libre service effectuent le plus grand nombre de repas en période diurne, avec deux pics, l'un en début et l'autre en fin de journée (Chase, Wangness et Baumgardt, 1976). En réalité les activités alimentaires sont rythmées par la distribution de nourriture, une ou deux fois par jour, parfois plus. En effet, la distribution de l'aliment incite l'animal à manger, même s'il n'a pas ingéré tout ce qui lui avait été distribué précédemment.

Dans le cas d'une distribution unique d'aliment le matin, Metz (1975), contrairement à Suzuki et Hidari (1973), observe que les vaches effectuent quand même un second repas avant la période d'obscurité. Lorsqu'il y a deux distributions par jour, les repas suivant ces distributions sont importants (" grands repas ») et durent entre 1 et 3 heures. Plusieurs petits repas (" repas intermédiaires ») les séparent au cours de la journée et de la nuit (3 à 8 en moyenne). La figure 1 (Baumont, non publié)
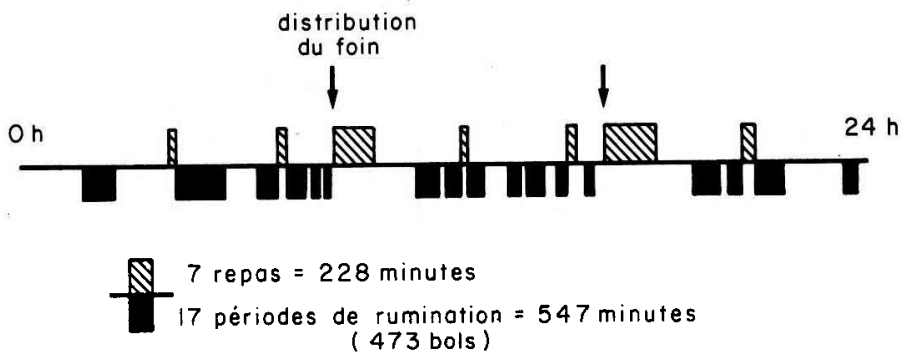

FIG. 1. - Séquence des activités alimentaires et méryciques chez un mouton recevant du foin de luzerne (Baumont, non publié).

montre que ces repas sont plus ou moins espacés et qu'entre eux s'intercalent une ou plusieurs périodes de rumination. Le temps passé à ruminer est proportionnellement plus élevé la nuit (cf. Dulphy, Rémond et Thériez, 1979 ; Jaster et Murphy, 1983), mais les périodes de rumination sont évidemment rythmées par la distribution de l'aliment (Murphy et al., 1983). A la suite d'un grand repas, la première période de rumination commence après une période d'inactivité masticatoire, ou temps de latence, de durée très variable (15 à $50 \mathrm{~min}$ ). 
Contrairement aux périodes d'ingestion, plus ou moins dispersées et de durée très variable, les périodes de rumination sont relativement bien individualisées. Leur durée, ainsi que l'intervalle de temps séparant deux périodes consécutives, sont rarement inférieurs à $20 \mathrm{~min}$. Le nombre des périodes de rumination est compris entre 10 et 17 par jour pour les fourrages ordinaires.

II existe des différences entre animaux quant à la durée et la répartition des activités alimentaires. Au pâturage, les vaches allaitantes passent de 5 à $12 \mathrm{~h}$ par jour à pâturer (Petit, 1972), les brebis de 3 à $13 \mathrm{~h}$ et les vaches laitières de 6 à $11 \mathrm{~h}$. A cela il faut ajouter 5 a $9 \mathrm{~h}$ de rumination pour les bovins et une durée équivalente pour les ovins. En stabulation, selon le type d'aliment, des béliers castrés passent en moyenne de 2 à $5,5 \mathrm{~h}$ par jour à ingérer et de 4 à $10 \mathrm{~h}$ à ruminer (I.N.R.A., 1978). A l'opposé, les vaches laitières passent de 5 à $10 \mathrm{~h}$ à ingérer et de 3,5 à 10,5 h à ruminer (tabl. 1).

\section{TABLEAU 1}

Activités journalières d'ingestion, de rumination et de mastication pour des vaches laitières. D'après Jarrige (in I.N.R.A., 1978).

\begin{tabular}{lcccc}
\hline & $\begin{array}{c}\text { Quantités de } \\
\text { matière sèche } \\
\text { ingérées } \mathrm{kg} / \mathrm{j}\end{array}$ & $\begin{array}{c}\text { Durée } \\
\text { d'ingestion } \\
\mathrm{min}\end{array}$ & $\begin{array}{c}\text { Durée de } \\
\text { rumination } \\
\text { min }\end{array}$ & $\begin{array}{c}\text { Durée unitaire } \\
\text { de mastication } \\
\text { min/kg de MS }\end{array}$ \\
\hline $\begin{array}{l}\text { Fourrage vert précoce } \\
\text { Fourrage vert tardif }\end{array}$ & 14,7 & 450 & 475 & 62 \\
Foin de luzerne & 10,4 & 562 & 622 & 113 \\
Foin de graminées & 12,7 & 426 & 562 & 78 \\
Concentré $+20 \%$ de foin & 12,3 & 513 & 576 & 88 \\
\hline
\end{tabular}
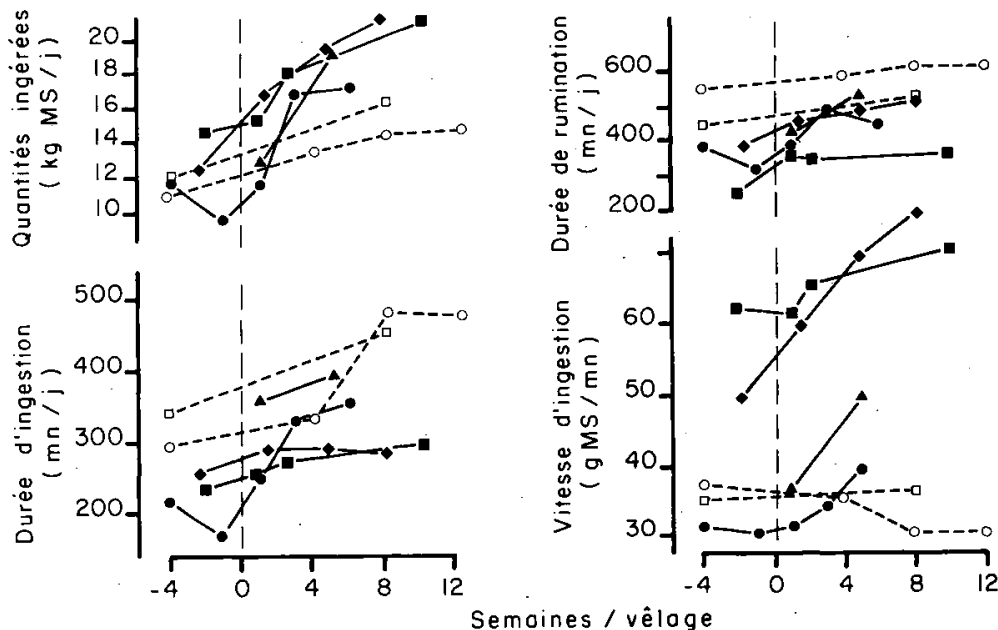

FIG. 2. - Evolution des paramètres du comportement alimentaire et des quantités ingérées en fin de gestation et début de lactation avec des rations riches en fibres (---) ( $\square$ Journet et Rémond, 1976; O Campling, 1966) ou très ingestibles (-) ( $\square$ Journet et Rémond, 1976 ; • Doreau et Rémond, 1982 ; $\Delta$ Coulon, 1981 ; • Faverdin, 1985). 
L'influence des besoins a été mise en évidence chez la vache laitière, pour laquelle on observe des variations importantes de quantités ingérées au cours du cycle de gestation-lactation (Campling, 1966 ; Journet et Rémond, 1976 ; Doreau et Rémond, 1982). L'accroissement des quantités ingérées en début de lactation semble d'autant plus élevé et rapide que la ration est plus ingestible (fig. 2). II résulte en général d'une augmentation de la durée d'ingestion. La vitesse moyenne d'ingestion s'accroît avec les rations fortement ingestibles (fig. 3 ; Faverdin, 1985). en même temps que les quantités ingérées, les vaches ont augmenté leur durée de rumination, la durée des grands repas, ainsi que le nombre et la durée des petits repas.

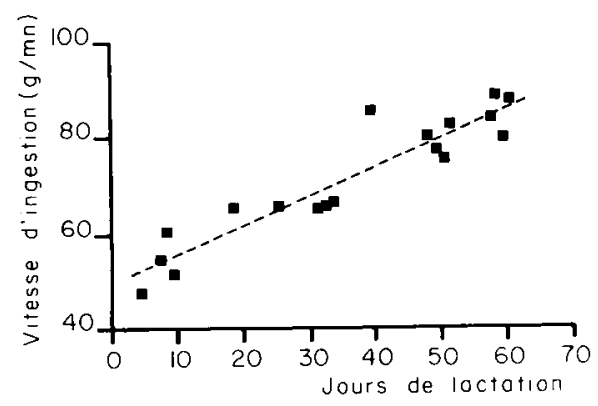

FIG. 3. - Evolution de la vitesse d'ingestion moyenne journalière avec le stade de lactation (VM $=50+0,6 \times$ jour, $R=0,93$ ) pour une vache recevant une ration mélangée $(60 \%$ ensilage de maïs, $40 \%$ aliments concentrés).

La comparaison entre 5 types d'animaux recevant les 3 mêmes fourrages (ensilage de maïs, foin précoce, foin tardif) est résumée dans le tableau 2 (Carle et Dulphy, 1980 ; Dulphy et Carle, 1986). Les grands repas sont relativement plus importants chez les béliers que les bovins (en proportion de la quantité journalière ingérée) et le début de la rumination est plus tardif que chez les vaches (54 contre 27 min après la fin du grand repas). Les brebis ont ingéré moins que les béliers lors des grands repas $(-19 \%)$; elles ont compensé ensuite en faisant plus de petits repas $(+1,9)$. Les chèvres ont ingéré des quantités de foin comparables à celles des béliers lors des grands repas, mais plus rapidement et ont effectué plus de petits repas. Enfin, les taurillons ont ingéré, lors des grands repas, des quantités bien plus faibles que les vaches, mais comparables si on les rapporte à celles ingérées par jour (33 et $31 \%$ ).

L'apport de concentré avant les deux grands repas de fourrages (tabl. 2) a entraîné une plus forte diminution du volume des grands repas de fourrage pour les chèvres.

Dans 11 comparaisons entre moutons et génisses recevant des fourrages verts (Dulphy et Michalet-Doreau, 1983) les animaux ont ingéré, par grands repas, des quantités de MS $/ \mathrm{kg} \mathrm{P}^{0,75}$ identiques en valeur absolue $(21-22 \mathrm{~g})$, mais différentes par rapport à celles ingérées par jour ( 30 et $24 \%$ respectivement) : les génisses ont eu tendance ensuite à faire plus de petits repas d'un volume relatif plus important que les moutons.

\section{II. - Les prises alimentaires.}

Chaque prise alimentaire (repas) est caractérisée par son " volume " (poids de matière fraîche ou sèche ingérée), sa durée et sa vitesse d'ingestion. 
1. - Etude cinétique.

La simple observation, puis l'enregistrement des mouvements de mastication (Ruckebusch, 1963) ont permis de faire de nombreuses mesures; les techniques modernes de dépouillement en ont amélioré la finesse d'analyse (Brun et Marzin, non publié). L'étude cinétique a pu être réalisée avec la mise en place de systèmes d'enregistrement en continu du poids de l'auge (Faverdin, 1985 ; Brun et Léveillé, non publié, fig. 4, issue des mêmes mesures que la fig. 1). Enfin, l'ajustement de

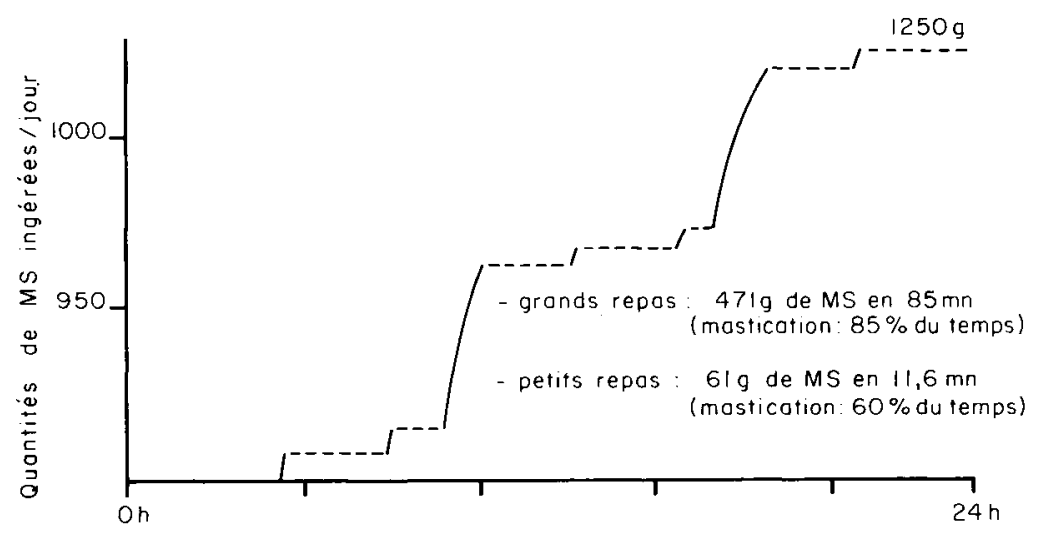

FIG. $\quad 4$.

l'évolution cumulée des quantités ingérées au cours des grands repas à une équation à 2 paramètres permet de calculer des vitesses d'ingestion instantanées théoriques, en particulier au début et à la fin des repas (fig. 5) et d'analyser aussi un grand nombre de repas.

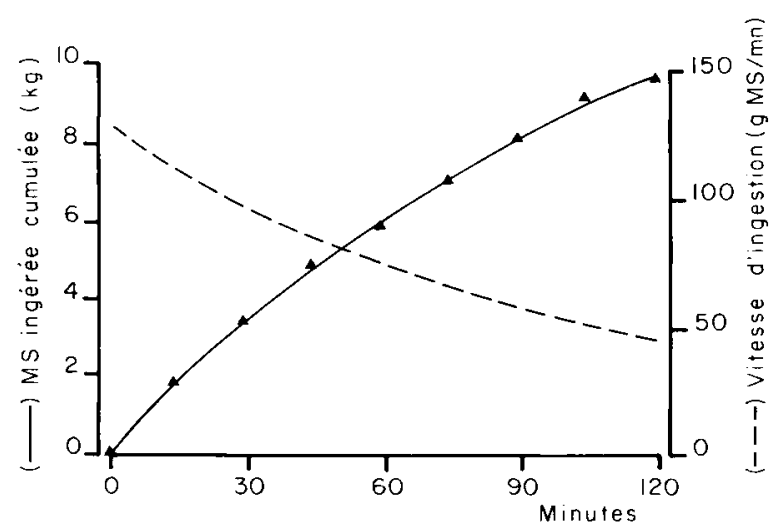

FIG. 5. - Exemple d'évolution des quantités ingérées cumulées ( $\mathbf{\Delta}$ ) au cours d'un grand repas par une vache en début de lactation et ajustées par l'équation :

$$
\text { QI }=14,8(1-\exp (-0,0087 \times t)) \text {. }
$$

La courbe de la vitesse d'ingestion (---) est obtenue par dérivation. 
Durant les périodes d'ingestion, les animaux ne mastiquent pas en permanence (Wangsness et al., 1976 ; Chase, Wangsness et Baumgart, 1976 ; Dulphy, Michalet-Doreau et Demarquilly, 1984). En effet, une partie du temps est consacrée a la perception olfactive et au tri de l'aliment. De plus, vers la fin du repas, l'animal prolonge les temps d'arrêt de mastication, probablement en raison d'un état de satiété qui se renforce. La mesure des vitesses d'ingestion peut donc aider à évaluer la motivation de l'animal pour l'aliment (goût, odeur) (cf. Kenney et Black, 1984) laquelle est fonction du degré de satiété.

La vitesse d'ingestion diminue constamment au cours du repas (Suzuki, Shinde et Hidari, 1973 ; Faverdin, 1985 ; Gill, Campling et Westgarth, 1966). On observe ce phénomène en suivant l'aire des " tracés masticatoires " (Cirio maisonnave, Boivin et Bost, 1981). Chez les vaches laitières, dont l'accès a une ration d'ensilage de maïs est limitée à 2 périodes de 4 heures, la vitesse initiale, calculée après ajustement de la courbe des quantités ingérées cumulées au cours du repas, est 4 à 8 fois supérieure à celle calculée en fin de repas. Le coefficient de variation de la vitesse d'ingestion initiale est beaucoup plus faible que celui de la vitesse finale (15\% contre 30 ). La répétabilité est améliorée par la limitation de l'accès à la ration (Faverdin, 1985).

En général, chez les moutons à l'entretien, les quantités ingérées lors des grands repas du matin et du soir sont identiques alors que les distributions d'aliments ne sont éloignés ici que de $8 \mathrm{~h}$ (Dulphy, non publié). Chez les vaches limitées à 2 périodes d'accès de $4 \mathrm{~h}$ à la ration, la durée du grand repas du soir est plus longue $(+17 \%)$ et les quantités ingérées plus élevées $(+18 \%)$ que lors du grand repas du matin (Faverdin, 1985). Or chez les vaches, les vitesses d'ingestion en fin de grand repas sont identiques matin et soir ( $33 \mathrm{~g}$ de $\mathrm{MS} / \mathrm{min}$ ) de sorte que l'on peut se demander si la sensibilité à l'état de replétion du rumen n'est pas modifiée par la prise d'aliments antérieure ou par des influences circadiennes.

La fin d'un repas correspond à une période d'incertitude pour le ruminant : la distribution de fourrage très appétible à la fin d'un grand repas entraîne une nouvelle prise alimentaire (Gatel, 1984). Nous avons observé le même phénomène en restituant à l'animal les refus d'un repas de fourrage vert très digestible.

La restriction des aliments augmente la vitesse d'ingestion et diminue le nombre de repas. La fréquence des coups de mâchoire pendant l'ingestion et la rumination, ainsi que le nombre de périodes de rumination ne sont pas modifiés (Freer, Campling et Balch, 1962 ; Freer et Campling, 1965 ; Gill, Campling et Westgarth, 1966, sur bovins ; Welch et Smith, 1969, sur ovins). La vitesse d'ingestion est d'autant plus élevée que la restriction alimentaire est forte. Elle peut être doublée pour de l'herbe déshydratée ou un aliment concentré (Freer et Campling, 1965). Les bols alimentaires ont un poids plus élevé, mais sont bien moins mastiqués, puisque le nombre de coups de mâchoire par bol est plus faible (Gill, Campling et Westgarth, 1966). La restriction du temps d'accès des animaux aux aliments provoque également un accroissement de la vitesse d'ingestion, laquelle ne suffit pas toujours à compenser le niveau d'ingestion. Les animaux ruminent ensuite plus longtemps (Freer, Campling et Balch, 1962 ; Suzuki, Shinde et Hidari, 1970).

Lorsque l'accès à l'auge est limité à 2 périodes de $4 \mathrm{~h}$ chez la vache laitière, la vitesse d'ingestion initiale des grands repas est plus élevée que dans le cas d'un 
accès permanent (153 contre $132 \mathrm{~g}$ de MS/min, Faverdin, 1985), mais la vitesse d'ingestion à la fin du repas n'est pas différente (38 contre $37 \mathrm{~g}$ de $\mathrm{MS} / \mathrm{min}$ ). L'apparition de la satiété au cours du grand repas ne semble donc pas directement liée à l'état de faim de l'animal au début du repas.

\section{2. - Grands repas.}

a) Variations liées aux animaux. Pour un même animal, il existe tout d'abord des variations du volume et de la vitesse d'ingestion des grands repas qui sont liées au stade physiologique des animaux et donc à leurs besoins, en particulier chez les vaches laitières (Faverdin, 1985). La vitesse initiale du grand repas augmente en début de lactation (semaines 1 à 9 après le vêlage) de même que les quantités ingérées au cours des grands repas ou de la journée. Cela indique que la vitesse d'ingestion, dès le début du repas, constitue un signe intéressant de l'état de faim, ou de l'appétit.

La vitesse d'ingestion varie également avant l'arrêt du repas, comme les quantités ingérées. Dans le même temps, le volume du rumen augmente, aussi bien chez la vache (Tulloh, 1966) que chez la brebis (Weekes, 1971). Si on admet que c'est l'encombrement par la ration des réservoirs digestifs qui constitue l'un des signaux de la satiété, le rapport de la vitesse d'ingestion avec la quantité ingérée constitue un bon indicateur que nous appellerons « vitesse relative ». Les vitesses relatives moyennes, en fin de repas, sont constantes entre la première et la neuvième semaine de lactation $(0,5 \% / \mathrm{min})$. II convient cependant de souligner qu'il existe une très grande variabilité entre jours et entre animaux.

II existe ensuite des différences entre individus dans la quantité ingérée par grand repas (qi) qui sont plus élevées que celles de la quantité journalière ingérée (QI) : coefficient de variation de $19,7 \%$ pour 21 fourrages distribués à des moutons (pour qi $=14,8 \mathrm{~g}$ de MS $/ \mathrm{kg} \mathrm{P}^{0,75}$ ) contre $10 \%$ (pour $\mathrm{Ol}=60 \mathrm{~g} \mathrm{de} \mathrm{MS} / \mathrm{kg} \mathrm{P}^{0,75}$ ) (Dulphy, non publié). Les coefficients obtenus sur des vaches laitières sont voisins (20\% pour qi contre $7,7 \%$ pour Ql, Faverdin, 1985).

II existe enfin des différences entre espèces dont nous avons parlé plus haut (tabl. 2).

b) Variations liées aux aliments. Les quantités ingérées de matière fraîche de fourrage vert lors des grands repas, chez le mouton, sont maximales et très élevées en début de cycle de végétation (jusqu'à $260 \mathrm{~g} / \mathrm{kg} \mathrm{P} \mathrm{P}^{0.75}$ ) lorsque le fourrage est riche en feuilles, en contenu cellulaire et en eau. II en est de même pour les bovins alors que les quantités ingérées de matière sèche (qi) varient peu en fonction du stade de végétation. Elles varient peu avec l'ingestibilité de la plante (QI) (Dulphy et Béchet, 1976) :

$$
q i=11,6+0,165 \text { Q }(r=0,44, n=50)
$$

Le pourcentage de la matière sèche totale ingérée lors des grands repas augmente donc lorsque la plante vieillit bien que, d'une plante à l'autre, les valeurs de qi soient très variables (tabl. 3 ).

Les quantités de matière sèche ingérées par grands repas sont plus faibles pour les foins que pour les fourrages verts initiaux (tabl. 3). La proportion ingérée, 


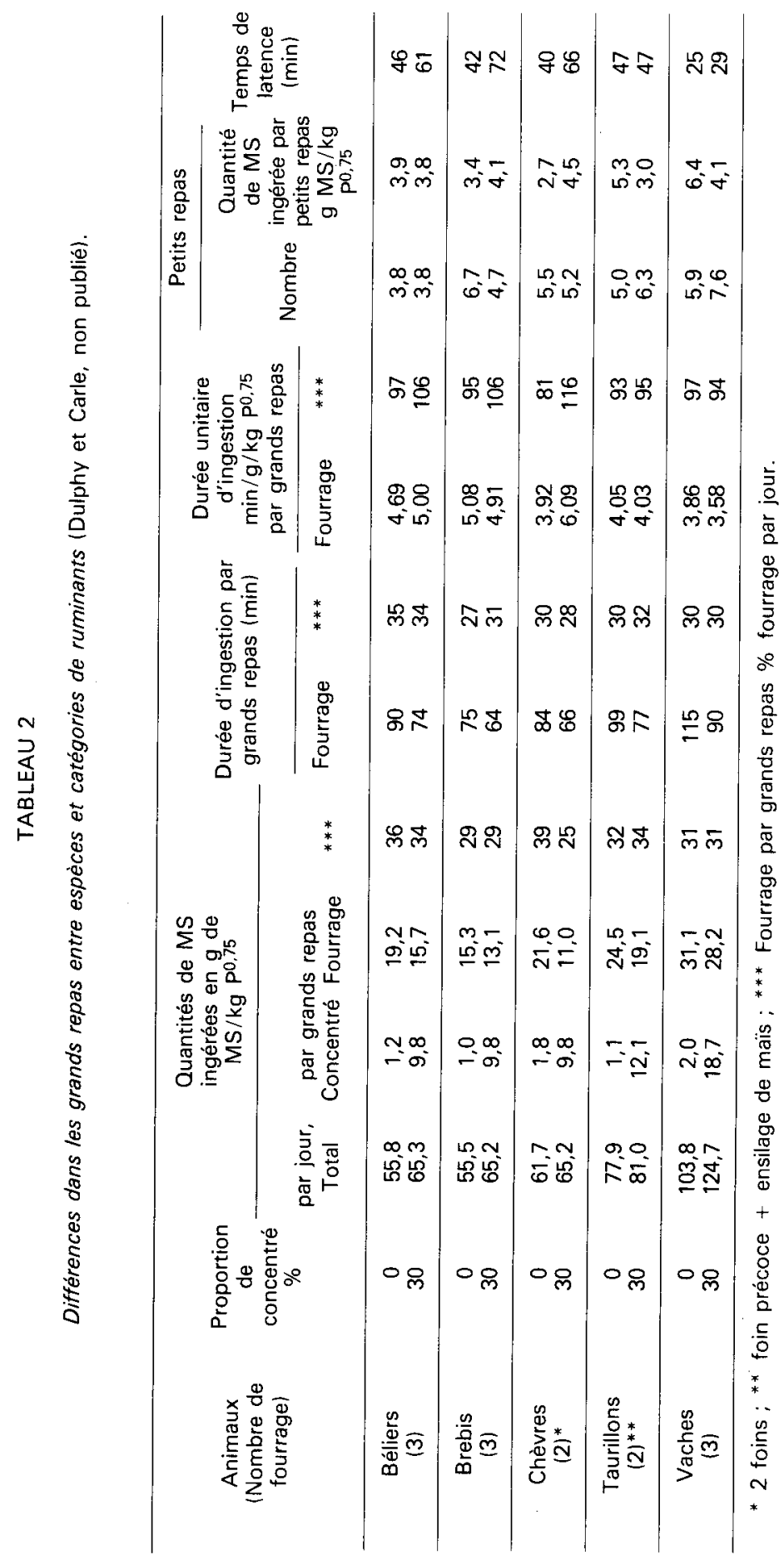


pour un grand repas, par rapport aux quantités ingérées par jour reste cependant égale à $32 \%$, c'est-à-dire comparable à la valeur de $35 \%$ obtenue pour les fourrages verts. Comme pour ces derniers, les valeurs de qi augmentent très peu avec l'ingestibilité des fourrages. Enfin, à même ingestibilité du fourrage, les moutons ingèrent plus de matière sèche sous forme de fourrage vert que de foin lors des grands repas ; cela pourrait être dû à une appétibilité supérieure du fourrage vert.

TABLEAU 3

Répartition des quantités de matière sèche ingérées par jour, entre les grands repas (2) et les petits repas chez le mouton (en $\mathrm{g}$ par $\mathrm{kg} \mathrm{P}^{0,75}$ ). (Dulphy, non publié).

\begin{tabular}{|c|c|c|c|c|c|}
\hline \multirow{2}{*}{\multicolumn{2}{|c|}{ Fourrages (n) }} & \multicolumn{3}{|c|}{ Quantités ingérées } & \multirow{2}{*}{$\begin{array}{c}\text { Nombre } \\
\text { de } \\
\text { petits } \\
\text { repas }\end{array}$} \\
\hline & & par jour & par grands & par petits & \\
\hline $\begin{array}{l}\text { Fourrages verts } \\
\text { - graminées } \\
\text { - légumineuses }\end{array}$ & $\begin{array}{l}(66) \\
(7)\end{array}$ & $\begin{array}{l}42-84 \\
77-95\end{array}$ & $\begin{array}{l}13-29 \\
27-34\end{array}$ & $\begin{array}{l}3,3 \\
3,4\end{array}$ & $\begin{array}{l}1-13 \\
5-9\end{array}$ \\
\hline Foins & ( 7) & 56,7 & 18,2 & 2,9 & 7,0 \\
\hline $\begin{array}{l}\text { Foins + fourrages } \\
\text { déshydratés }\end{array}$ & (38) & 54,5 & 17,5 & 2,6 & 7,4 \\
\hline Fourrages verts initiaux & & 65,5 & 23,0 & 2,4 & 8,0 \\
\hline $\begin{array}{l}\text { Ensilages d'herbe } \\
\text { Fourrages verts initiaux }\end{array}$ & (63) & $\begin{array}{l}51,4 \\
72,5\end{array}$ & $\begin{array}{l}12,7 \\
26,1\end{array}$ & $\begin{array}{l}3,5 \\
3,7\end{array}$ & $\begin{array}{l}7,5 \\
\mathbf{5 , 5}\end{array}$ \\
\hline Paille + concentré & (3) & $\begin{array}{r}27,6(P) \\
+13,2(C)\end{array}$ & $\begin{array}{r}10,0(P) \\
+\quad 6,6(C)\end{array}$ & $\begin{array}{c}3,0(P) \\
-\end{array}$ & 2,5 \\
\hline
\end{tabular}

Pour les ensilages d'herbe bien conservés (Dulphy, Michalet-Doreau et Demarquilly, 1984 ; Dulphy, 1985), les quantités ingérées par grands repas correspondent à $25 \%$ seulement de la quantité totale ingérée par $24 \mathrm{~h}$ contre $36 \%$ pour les fourrages verts initiaux. A même ingestibilité que pour les fourrages verts, les moutons ingèrent alors deux fois moins de matière sèche lors de ces grands repas. Les ensilages de légumineuses sont cependant mieux ingérés que ceux de graminées, quoiqu'en même proportion des quantités journalières ingérées. Lorsque la qualité de conservation des ensilages se dégrade, les valeurs de qi diminuent lentement. Ceci est encore plus net lorsque les ensilages sont récoltés en brins longs. Tout se passe alors comme si l'animal anticipait sur les difficultés qui l'attendent pour ruminer (cf. aussi Deswysen, 1980). Les génisses ne réagissent pas de la même façon et ingèrent, lors des grands repas, autant d'ensilage de bonne qualité que de fourrage vert. Ces quantités baissent légèrement lorsque I'ensilage n'est pas haché et fortement lorsqu'il est mal conservé (Dulphy, Michalet-Doreau et Demarquilly, 1984).

Pour 3 pailles (Dulphy, non publié) les quantités ingérées par grands repas ont été faibles, mais égales encore à $36 \%$ des quantités journalières ingérées.

L'apport de concentré, avant la distribution d'un fourrage, diminue la valeur de qi. Pour les 3 fourrages présentés plus haut (tabl. 2), l'apport de concentré a 
fait baisser cette valeur pour tous les ruminants. A l'exception des chèvres, les rapports qi/QI de fourrage n'ont cependant pas été modifiés. II pourrait $y$ avoir aussi des différences selon la nature du concentré, comme c'est le cas pour la mélasse dont l'apport diminue la durée des grands repas de fourrage plus que pour celui de la pulpe de betterave déshydratée ou de maïs (Guérin et Dulphy, 1984).

\section{3. - Petits repas.}

Le nombre et le volume des petits repas sont variables selon les animaux et les aliments. Pour les fourrages verts, le nombre de petits repas est très élevé en début de cycle de végétation (Dulphy et Béchet, 1976) ; il baisse lorsque la plante vieillit. La diminution d'ingestibilité au cours du cycle de végétation s'explique alors un peu par la baisse de la quantité ingérée lors des grands repas, mais surtout par la baisse des quantités ingérées lors des petits repas, dont le nombre, plus que la durée, est modifié. D'une espèce fourragère à l'autre il existe de grandes variations du nombre des petits repas. Par exemple, à même digestibilité, le nombre de petits repas est plus élevé pour le ray-grass d'ltalie et la luzerne que pour les autres graminées et il augmente lorsque la plante est plus riche en eau.

Avec les ensilages d'herbe, les moutons compensent partiellement leur faible ingestion lors des grands repas, par rapport aux fourrages verts originels, par un nombre plus élevé de petits repas (Dulphy, Michalet-Doreau et Demarquilly, 1984). Avec les pailles, le nombre de petits repas est faible (tabl. 3) bien que leur volume reste comparable à celui des autres fourrages. L'apport de concentré à base de céréales et de tourteau ne semble pas modifier le nombre et le volume des repas intermédiaires (tabl. 2). En revanche, lorsque le concentré est de la mélasse (Guérin et Dulphy, 1984), le temps de latence après les grands repas de fourrage, ainsi que le nombre de petits repas augmentent.

\section{III. - Phénomènes associés à l’ingestion.}

Les. principaux phénomènes accompagnant les repas ou induits par eux et impliqués dans le contrôle des quantités ingérées sont digestifs, métaboliques et hormonaux. On observe, lors des prises alimentaires, des modifications de la motricité des pré-estomacs, de la structure de leur contenu, ainsi que des modifications du contenu digestif par la salivation et la digestion. Ces modifications, ainsi que la rumination, sont importantes à considérer car elles jouent un double rôle, d'une part en facilitant la prise de nourriture, d'autre part en limitant cette prise lorsque sont générés certains signaux entraînant l'arrêt du repas.

\section{1. - Phénomènes digestifs et méryciques.}

a) Motricité des pré-estomacs. A chaque type de comportement alimentaire ou mérycique sont associés des profils moteurs particuliers des pré-estomacs (Ruckebusch et Buéno, 1972). Plusieurs articles de synthèse ont été consacrés à la 
motricité du réticulo-rumen (Reid, 1963 ; Wyburn, 1979 ; Ruckebusch, Buéno et Fioramonti, 1981).

Pendant la prise d'aliments, les contractions du réticulo-rumen sont plus fréquentes que pendant les périodes de repos (Balch, 1958 ; Freer, Campling et Balch, 1962 ; Reid, 1963 ; Waghorn et Reid, 1977, tabl. 4). L'accélération marquée pendant le premier tiers du repas semble en relation avec l'appétence de l'animal pour l'aliment. La fréquence des contractions réticulaires est liée à l'aire des " tracés masticatoires " (fréquence $x$ amplitude des coups de mâchoires ; Cirio Maisonnave, Boivin et Bost, 1981). La prise de nourriture est également accompagnée d'une augmentation de l'amplitude des contractions (Smith, Weeks et Jarrard, 1969) en particulier de la deuxième phase de contraction. L'accélération de la motricité accroit le débit réticulo-omasal au cours du repas et autorise, au cours des grands repas, une ingestion plus élevée que ne le permettrait une vitesse de vidange normale du rumen (Grenet, 1987). Cette accélération, d'autant plus forte que les aliments sont appétibles, (Reid, 1963) explique le volume élevé des grands repas pour certains fourrages verts ou les foins bien conservés.

TABLEAU 4

Influence de la prise alimentaire sur la motricité du réticulo-rumen (contractions/min)

\begin{tabular}{|c|c|c|c|c|c|c|}
\hline Auteurs & Animaux & Rations & $\begin{array}{l}\text { Lors du } \\
\text { début }\end{array}$ & $\begin{array}{l}\text { repas } \\
\text { fin }\end{array}$ & Rumination & Repos \\
\hline Balch, 1958 & Vaches & $\begin{array}{l}\text { foin } \\
\text { concentré }+ \text { foin } \\
\begin{aligned} \text { concentré }+ \text { betteraves } \\
\quad+\text { foin }\end{aligned}\end{array}$ & $\begin{array}{l}1,6 \\
1,9 \\
1,8\end{array}$ & $\begin{array}{l}1,4 \\
1,3 \\
1,4\end{array}$ & & \\
\hline Freer et al., 1962 & Vaches & $\begin{array}{l}\text { foin } \\
\text { paille }\end{array}$ & $\begin{array}{l}1,4 \\
1,4\end{array}$ & & $\begin{array}{l}1,06 \\
0,91\end{array}$ & $\begin{array}{l}1,25 \\
1,19\end{array}$ \\
\hline Waghorn et Reid, 1977 & $\begin{array}{l}\text { Mouton } \\
\text { Bovin }\end{array}$ & $\begin{array}{l}\text { foin de luzerne } \\
\text { ray grass vert } \\
\text { foin de luzerne } \\
\text { ray grass vert }\end{array}$ & $\begin{array}{l}2,6 \\
2,6 \\
2,3 \\
2,2\end{array}$ & & $\begin{array}{l}1,57 \\
1,88 \\
1,54 \\
1,67\end{array}$ & $\begin{array}{l}1,30 \\
1,36 \\
1,68 \\
1,70\end{array}$ \\
\hline
\end{tabular}

Les bols alimentaires arrivant dans le réseau n'y séjournent pas et sont projetés avec force vers l'arrière (Waghorn et Reid, 1977 ; Wyburn, 1979 ; Deswysen et Ehrlein, 1981). Ils s'accumulent dans la partie haute du rumen et se désagrègent sous l'effet des contractions très puissantes du rumen, grâce aussi à l'importance de la phase liquide ( 88 à $90 \%$ du contenu). Le circuit des particules dans le réticulorumen est le suivant : après un premier tri durant lequel les plus fines particules tombent directement dans le réseau, les plus grosses, de faible densité, passent dans le sac crânial et les mouvements du réticulo-rumen entraînent la stratification des particules selon leur densité. Cette stratification est très nette chez les bovins ; elle permet aux grosses particules d'être " aspirées " lors de la rumination.

b) Contenu ruminal. La quantité du contenu digestif chez les ruminants est élevée (cf. I.N.R.A., 1978). Elle varie de 11 à $21 \%$ du poids vif pour des bovins à 
l'engrais, la majeure partie étant présente dans le réticulo-rumen : 10 à $16 \%$ du poids vif (tabl. 5).

\section{TABLEAU 5}

Poids de contenu frais et sec dans le réticulo-rumen de bovins de $500 \mathrm{~kg}$. (d'après Jarrige, in I.N.R.A., 1978.)

\begin{tabular}{lcccc}
\hline \multicolumn{1}{c}{ Ration } & $\begin{array}{c}\text { Quantité de } \\
\text { contenu } \\
\text { frais } \\
(\mathrm{kg})\end{array}$ & $\begin{array}{c}\text { Teneur } \\
\text { en MS } \\
(\%)\end{array}$ & $\begin{array}{c}\text { Quantité de } \\
\text { contenu } \\
\text { sec } \\
(\mathrm{kg})\end{array}$ & $\begin{array}{c}\text { Quantités de } \\
\text { matière sèche } \\
\text { ingérées } \\
(\mathrm{kg})\end{array}$ \\
\hline $\begin{array}{l}\text { Fourrage vert } \\
\quad \text { précoce }\end{array}$ & 49 & 10,3 & & \\
$\quad-$ tardif & 60 & 11,8 & 5,0 & 11,7 \\
$\begin{array}{l}\text { Foin de luzerne } \\
\text { Foin de graminées }\end{array}$ & 62 & 14,8 & 7,1 & 10,1 \\
Foin de graminées & 82 & 14,7 & 9,2 & 9,2 \\
$\quad+30 \%$ orge & 61 & 15,5 & 9,1 & 9,0 \\
Concentré $+20 \%$ de foin & 51 & 13,1 & 6,5 & 9,4 \\
\hline
\end{tabular}

La masse du contenu ruminal, qui contient seulement 10 à $15 \%$ de matière sèche, augmente pendant les grands repas (Campling, Freer et Balch, 1961 ; Journet et Jarrige, 1970), la quantité de matière sèche augmentant proportionnellement plus que la quantité de liquide (Baile, 1979). Cette masse, qui est donc maximale après le grand repas du soir (Baich, 1958 ; Dulphy, Béchet et Thomson, 1975 ; Thomson et al., 1985) peut varier au cours de la journée de façon importante dans le cas de I'herbe jeune (Paquay, De Baere et Lousse, 1971). Pour des vaches de petit format Tulloh, Hugues et Newth (1965) trouvent des variations de contenu allant de 30 (minimum) à 45 litres (maximum) au cours d'une journée de pâturage.

Pour un animal donné, le contenu, mesuré toujours à la même heure après le repas, varie dans le même sens que les quantités ingérées (Piton, 1975) et donc selon les besoins de l'animal (Tulloh et Hugues, 1965 ; Pond et al., 1984).

L'effet du type de ration sur la quantité de contenu en fin de repas a été mesuré en de multiples circonstances depuis les travaux de Blaxter, Wainman et Wilson (1961) et de Campling, Freer et Balch (1961). Ainsi, elle est plus importante avec un foin qu'avec une paille non supplémentée ; elle augmente dans le cas de l'addition d'urée à la paille (Campling, Freer et Balch, 1962), et baisse dans le cas du broyage des foins (Campling, Freer et Balch, 1963) : elle baisse si la proportion de concentré dans la ration augmente (Bines et Davey, 1970). Cependant, l'amplitude des variations est souvent faible (Thornton et Minson, 1972 et 1973 ; Laredo et Minson, 1973), même pour des fourrages très différents, en l'absence de facteurs limitants tels que l'inappétibilité de l'aliment, une carence en azote ou un excès de concentré dans la ration. Dans le cas d'ensilages mal conservés ou à brins longs, le niveau des contenus à la fin des grands repas est plus faible que pour les ensilages de bonne qualité (Dulphy, Béchet et Thomson, 1975).

Soulignons par ailleurs que la consistance du contenu de rumen évolue au cours des repas (Hidari, 1979). La stimulation mécanique de la paroi ruminale qu'elle 
entraîne pourrait être un signal de l'arrêt du repas, au même titre que le volume du contenu, lequel augmente plus vite que la masse en cours de repas.

Le contenu du réticulo-rumen ne passe à travers l'orifice réticulo-omasal que si les particules alimentaires sont suffisamment dégradées. La quantité de matière sèche qui passe par unité de temps dépend de deux facteurs : (1) l'état du contenu réticulo-omasal, les éléments les plus fins passant le plus facilement grâce à leur densité élevée ; (2) le gradient de pression entre le réticulo-rumen et le feuillet. Celui-ci n'est positif que lors de la contraction d'une partie quelconque du réticulorumen, c'est-à-dire durant 30 à $40 \%$ du temps environ (Buéno et Ruckebusch, 1974).

Le contenu réticulaire qui franchit l'orifice réticulo-omasal est composé d'une partie liquide, dont le débit augmente au cours de la prise alimentaire, pour reprendre sa valeur initiale une heure environ après le début du repas et d'éléments solides, dont la vitesse de passage diminue linéairement pendant I'heure qui suit le début du repas, puis augmente progressivement jusqu'au suivant (Alderson et al., 1972).

c) Modifications chimiques du contenu digestif. Au fur et à mesure que le ruminant ingère son repas, la quantité de salive sécrétée s'accroît de 170 à $430 \mathrm{ml} / \mathrm{min}$ chez une vache (Balch, 1958 ; Bailey 1961 ; Bailey et Balch, 1961a et $1961 b$; Wilson, 1963 ; Wilson et Tribe, 1963 ; Orth et Kaufmann, 1966). La salive apporte 70 à $90 \%$ des liquides contenus dans le rumen et le rythme de sécrétion est encore accru lors des périodes de rumination (Kaufmann et Orth, 1966 ; Lawlor, Giesecke et Walser-Kärst, 1966). Le pH de la salive est proche de 8,6. Sa richesse en ions $\mathrm{Na}^{+}$et $\mathrm{K}^{+}$ainsi qu'en bicarbonates et en phosphates permet de tamponner efficacement le contenu du rumen. Son rôle est important sur le plan physique : aide à la mastication, abaissement de la tension superficielle du contenu de rumen, ce qui évite la formation de mousse. La salivation contrôle aussi la consistance du contenu. Si on l'empêche (Ruckebusch, Buéno et Fioramonti, 1981), la masse du contenu devient compacte et toute rumination est impossible.

L'intensité de la sécrétion salivaire varie avec le type d'aliment, selon la structure, la teneur en matière sèche et la vitesse d'ingestion (Balch, $1958 ;$-Lawlor, Giesecke et Walser-Kärst, 1966) ; Orth et Kaufmann, 1966 ; Ruckebusch, Buéno et Fioramonti, 1981). Ainsi au cours du repas baisse-t-elle quand on distribue un foin long à la place d'un foin broyé (Ruckebusch, Buéno et Fioramonti, 1981). Ce phénomène est amplifié au cours de la journée (Lawlor, Giesecke et Walser-Kärst, 1966). La sécrétion salivaire par minute est augmentée pour un aliment concentré (Balch, 1958), mais la sécrétion totale, rapportée au gramme de matière sèche ingérée est diminuée. La quantité de salive sécrétée pour les fourrages verts est proche de celle sécrétée pour des foins.

Enfin, au cours du repas, la pression osmotique du contenu de rumen augmente (Scott, 1975), ainsi que la concentration en acides gras volatils.

d) Rumination. La rumination, dont la durée par nycthémère excède souvent le temps d'ingestion, a pour effet d'abaisser la taille des particules alimentaires et de permettre à celles-ci de franchir l'orifice réticulo-omasal (Pearce et Moir, 1964 ; Ulyat et al., 1986). En effet, la rumination est associée à une sécrétion salivaire et une motricité accrues par rapport aux périodes de repos. Ces phénomènes 
améliorent le tri des particules alimentaires dans le réticulo-rumen (Wyburn, 1979) et facilitent, entre les repas, le transit des petites particules vers le feuillet. Le déterminisme de la rumination a été étudié par Ruckebusch (1963), Ruckebusch et Laplace (1967), Gordon (1968), Gordon et Mc Allister (1970), Welch (1982), Kennedy (1985). Sa durée totale ne semble pas pouvoir dépasser un plafond de $600 \mathrm{~min} /$ jour chez le mouton à l'entretien (Dulphy, non publié) et 650 min environ chez la vache laitière. Ce plafond peut être le facteur limitant de l'ingestion des fourrages difficiles à réduire en fines particules. Au-dessous de ce plafond, la durée de rumination varie avec la quantité de parois végétales ingérées et leur digestibilité, ainsi qu'avec le degré de hachage ou de broyage de l'aliment (Dulphy, Rémond et Thériez, 1979).

e) Rôle des phénomènes digestifs associés. La régulation des quantités d'énergie passe par celle de la quantité d'aliments ingérée et, à court terme, celle des quantités ingérées lors de chacun des repas (Baile, 1975 ; Forbes, 1977 a et b, 1980). La régulation à long terme permet ensuite de corriger d'éventuelles imprécisions dans l'ajustement des quantités journalières ingérées.

Dans leur synthèse datant de 1962, Balch et Campling insistaient déjà sur le nombre élevé de facteurs de contrôle et la complexité des mécanismes d'action, mais surtout sur le rôle fondamental de la réplétion du réticulo-rumen lié au séjour prolongé et obligatoire des aliments dans ce compartiment digestif. En 1964 Conrad, Pratt et Hibbs, puis en 1965 Montgomery et Baumgardt ont classé les rations en deux catégories, celles de faible valeur nutritive dont l'ingestion est réglée par l'encombrement du réticulo-rumen et celles de valeur nutritive élevée dont I'ingestion est réglée par des mécanismes chémostatiques et thermostatiques. Les rations du premier type seront donc celles dont la régulation de l'ingestion est directement dépendante des phénomènes digestifs.

La distension de la paroi digestive est, depuis longtemps considérée comme étant à l'origine de signaux entraînant la fin du repas chez le ruminant consommant des rations à base de fourrages. Des récepteurs sensibles à la pression ou à la distension des pré-estomacs (Iggo, 1960; Leek, 1969), en particulier lors de surcharge du rumen (Campling et Balch, 1961; Hidari, 1984) expliquent l'influence de l'état de réplétion du rumen dont la définition est donnée par ailleurs par Jarrige (in I.N.R.A., 1978). Cet état de réplétion est lié à l'encombrement des pré-estomacs par la masse alimentaire, et surtout les parois végétales, en cours de dégradation. Grovum (1979) estime cependant que le rôle du réseau serait plus important que celui du rumen dans le cas d'aliments broyés. Comme le rumen et le réseau communiquent largement, l'encombrement de l'un doit cependant se répercuter sur l'autre.

Les observations suivantes indiquent que le rôle de l'état de réplétion du réticulo-rumen a été parfois surestimé. Les quantités de matière fraîche ingérées lors des grands repas de fourrages verts sont maximales en début de cycle de végétation, période au cours de laquelle le nombre de repas spontanés est élevé et le volume du contenu de rumen plus élevé qu'en fin de cycle de végétation. II est vrai que le fourrage, alors très aqueux et digestible, encombre peu de temps le rumen. L'animal peut alors augmenter le nombre de ses repas et la quantité de 
matière sèche ingérée. Cette compensation n'intervient plus lorsque les fourrages sont trop riches en eau (Vérité et Journet, 1970). Les quantités de matière sèche ingérées par grands repas varient relativement peu en fonction du stade de végétation et tout se passe comme si leur effet d'encombrement était lié à la matière sèche seule. Le ruminant est donc capable de tolérer temporairement un état de distension élevé de ses pré-estomacs avec des aliments très aqueux qui sont digérés rapidement.

Les pailles distribuées seules sont ingérées en quantité faible en raison de leur charge en parois lignifiées mais aussi de leur faible appétibilité (Greenhalgh et Reid, 1971 ) et de leur carence en azote. Les animaux n'utilisent pas alors toute la capacité du réticulo-rumen (Campling, Freer et Balch, 1961) ; Ils le font lorsque I'on apporte de l'urée (Campling, Freer et Balch, 1962). Par ailleurs, I'animal augmente progressivement son ingestion à long terme (Xandé, 1978), ce qui peut résulter à la fois d'une accoutumance sensorielle, d'une diminution des réserves adipeuses et, aussi, d'une adaptation digestive. La notion d'encombrement du réticulo-rumen n'est donc pas rigide. Contrairement à ce que prévoit le modèle de Forbes (1980) (beaucoup de petits repas pour les fourrages pauvres), l'animal fait peu de repas ; ce pourrait être dû à la fois à la très faible appétibilité des pailles et à la nécessité de ruminer non prise en compte par l'auteur.

Avec les ensilages d'herbe, surtout chez le mouton, le rôle de l'encombrement peut être secondaire (Dulphy, Béchet et Thomson, 1975) par rapport aux autres phénomènes sensoriels, métaboliques ou mécaniques limitant la quantité ingérée par grands repas (Dulphy, 1985). Il en est de même avec les rations riches en aliment concentré (Bines et Davey, 1970) bien qu'un certain encombrement du réseau existe lors de l'ingestion de ces aliments (Campling et Freer, 1962 ; Freer et Campling, 1963).

La quantité du contenu de rumen est plus élevée le soir que le matin, au pâturage alors que l'ingestion est lente (Thomson et al., 1985) mais aussi à l'auge. Les ruminants ne réagissent donc pas de la même façon à l'état de distension du réticulo-rumen selon le moment de la journée, ce dont le modèle de Forbes ne tient pas compte non plus. Enfin le niveau atteint à la fin d'un grand repas peut être modifié en redonnant à l'animal un fourrage très appétible (Gatel, 1984). Il est aussi variable avec l'état nutritionnel azoté de l'animal (Egan, 1970).

Le ruminant peut donc cesser son ingestion avant que son rumen n'ait atteint la limite maximal de remplissage pour les causes suivantes : faible appétibilité de I'aliment, carence en azote, effets spécifiques de l'aliment sur la motricité, nécessité de ruminer, augmentation de la pression osmotique dans le réticulo-rumen (Ternouth et Beattie, 1971), libération d'acides dans le rumen, sécrétions hormonales.

Soulignons aussi que la limitation prioritaire par la distension concerne les rations dans lesquelles les fourrages sont exclusifs ou dominants. Ce n'est plus le cas avec les rations riches en énergie qui couvrent les besoins de l'animal sans réplétion maximale. Enfin, une analyse fine de ce qui se passe avec un temps d'accès limité à l'aliment serait nécessaire pour apprécier la quantité maximale du contenu digestif fixée par la taille de l'animal et ses réserves adipeuses. 


\section{2. - Modifications humorales liées à l'ingestion.}

La prise d'aliments est à l'origine de la fourniture d'énergie aux cellules et de processus anaboliques avec mise en réserve de l'excès des métabolites. Les modifications du sang consécutives au repas interviennent dans la régulation de l'ingestion comme l'avaient montré Seoane, Baile et Martin (1972) avec des moutons affamés dont l'ingestion était diminuée après mise en circulation croisée avec des congénères rassasiés. L'information ainsi véhiculée par voie sanguine peut provenir : (1) de l'absorption des produits terminaux de la digestion, (2) de la production ou de l'utilisation de métabolites, notamment au niveau du foie et du système nerveux, (3) de sécrétions des glandes endocrines.

Des points particuliers du contrôle humoral des quantités ingérées à court terme seront discutés ici par rapport aux principales modifications humorales associées à la prise d'aliments, les autres aspects étant généralement déjà explicités dans les revues de Balch et Campling, 1962 ; Jones, 1972 ; Baile et Forbes, 1974 ; Gallouin et Focant, 1980 ; Forbes, 1980 : Baile, Della-Fera et McLaughlin, 1983.

\section{a) Modifications métaboliques.}

Au cours du grand repas, le débit sanguin veineux portal augmente de 30 à $50 \%$ en 90 min (Webster, 1974 ; Huntington, 1982). L'augmentation de ce débit est positivement corrélée avec l'absorption d'acides gras volatils (AGV) chez le mouton, en particulier du propionate (Weekes et Webster, 1975). Chez la vache laitière, il existe une relation positive entre les quantités d'énergie métabolisable ingérées et le débit de la veine porte (Lomax et Baird, 1983).

L'augmentation de la production de chaleur lors des repas lou des périodes de rumination) atteint 40 à $80 \%$ en début de repas chez le mouton et elle peut se poursuivre pendant la totalité du repas (revue de Webster, 1980). L'élévation concomitante du rythme cardiaque n'est pas liée à un état d'excitation de l'animal puisque les béta-bloquants sont sans effet ; elle n'est pas liée davantage à la présence d'aliments dans le rumen, car leur introduction directe par une canule ne provoque qu'une réponse 12 à 50 fois plus faible que celle observée lors d'un repas normal (Osuji, Gordon et Webster, 1975). La production de chaleur par $\mathrm{kg}$ de matière sèche ingérée est d'autant plus importante que la vitesse d'ingestion est faible (Holmes, McLean et Lockyer, 1978). La température hypothalamique varie peu avec la prise alimentaire et les modifications de production de chaleur ne constituent donc probablement pas un signal majeur de satiété (revue de Baile et Forbes, 1974).

L'absorption accrue des AGV et de leurs dérivés métaboliques constitue l'essentiel des modifications nutritionnelles rencontrées au cours des grands repas. Les remaniements par l'épithélium du rumen des AGV sont à l'origine de l'augmentation de la concentration dans le sang des corps cétoniques ( $\beta$-hydroxybutyrate et acétoacétate), du lactate et du pyruvate (revue de Stevens, 1970). Au cours du repas, l'absorption nette d'AGV augmente de près de 80 à $90 \%$ par rapport au début du repas (Huntington, 1982). La concentration de l'acétate plasmatique est maximale entre 3 et $6 \mathrm{~h}$ après le début du repas, parallèlement à celle des AGV dans le rumen. L'évolution des AGV est analogue dans le sang veineux jugulaire et portal 
(Simkins, Suttie et Baumgardt, 1965 ; Bines et Davey, 1978) avec cependant une faible amplitude de variation pour le propionate et le butyrate au niveau jugulaire du fait de leur captation et transformation par le foie (De Jong, 1981a). Les cinétiques de concentration des corps cétoniques sont voisines de celles de l'acétate (Bines et Davey, 1978 ; Bines, Hart et Morant, 1983 ; Faverdin, 1985 ; De Jong, 1981).

Au cours des petits repas spontanés, les concentrations des AGV dans le sang veineux, aussi bien jugulaire que portal, ne semblent pas modifiées par la prise d'aliments (De Jong, 1981b ; Chase, Wangsness et Martin, 1977), exception faite du butyrate et du $\beta$-hydroxybutyrate dont les concentrations plasmatiques au niveau de la veine porte augmentent après 10 à $15 \mathrm{~min}$.

Malgré les nombreux essais de perfusion d'AGV dans le rumen et dans le sang, leur rôle dans le contrôle de la satiété reste discutable. La principale raison semble être d'ordre méthodologique : la simulation de la production d'AGV au cours du repas par des perfusions est difficile à réaliser dans des conditions physiologiques de $\mathrm{pH}$, de pression osmotique... Aucun effet net d'un mélange d'AGV sur la prise alimentaire à court terme n'a été obtenu (Baile et Mayer, 1968 ; Focant et Gallouin, 1978 ; De Jong, 1981c), contrairement à la diminution d'ingestion observée avec des doses élevées d'acétate (revue de Gallouin et Focant, 1980).

Le propionate et le lactate sont métabolisés en glucose par le foie, la néoglycogenèse hépatique mesurée chez la vache en lactation étant de l'ordre de 6 à $7 \mathrm{mmoles} / \mathrm{min}$ (Lomax et Baird, 1983). Cette synthèse importante, associée à une faible quantité de glucose absorbée au niveau digestif avec les régimes sans amidon fait apparaître une absorption nette négative du glucose en cours de repas (Huntington, 1982). Cependant, la glycémie du sang veineux jugulaire est stable (Bines, Hart et Morant, 1983) ou diminue au cours du grand repas (Bassett, 1974 ; De Jong, 1981a ; Faverdin, 1985) ; elle ne reflète donc pas cette arrivée de glucose dans l'organisme. En fait, l'utilisation tissulaire du glucose est vraisemblablement accrue par la sécrétion d'insuline stimulée par la prise alimentaire. Au cours des petits repas, la glycémie semble peu affectée (De Jong, 1981b), sauf si ceux-ci dépassent une vingtaine de minutes (Chase, Wangsness et Martin, 1977).

Les injections de glucose dans la carotide chez le mouton provoquent une diminution des quantités ingérées (Baile, 1971) et les injections de 2-déoxy-glucose, qui entraînent une glucoprivation, stimulent la prise alimentaire (Houpt, 1974). En revanche, dans d'autres essais, des infusions de glucose n'ont provoqué aucune modification de la quantité d'aliments ingérée (revue de Baile et Forbes, 1974), ce qui laisse supposer des différences importantes avec les espèces monogastriques (revue de Le Magnen, 1970). La faible absorption de glucose au cours du repas et sa production hépatique ont sans doute peu d'incidence dans le contrôle de la prise d'aliments chez les ruminants.

Les concentrations plasmatiques d'acides aminés (Anderson, Mangan et Wright, 1974) et d'azote $\alpha$-aminé (Bassett, 1974 ; Faverdin, 1985) diminuent légèrement pendant et à la suite $(2 \mathrm{~h})$ du grand repas. Comme pour le glucose, ces variations ne semblent pas liées aux flux métaboliques et il est donc difficile de connaître le rôle des acides aminés dans l'apparition de la satiété.

La concentration des acides gras non estérifiés plasmatiques diminue de près de $2 / 3$ au cours du grand repas et des 2 h qui suivent quand elles sont élevées parce 
que l'animal est amené à mobiliser ses réserves corporelles, comme le font par exemple les vaches en début de lactation (Decaen et Journet, 1967 ; Bines, Hart et Morant, 1983 ; Faverdin, 1985). Cette diminution est faible, voire absente lorsque les besoins sont limités : génisses (Simkins, Suttie et Baumgardt, 1965), vaches en fin de gestation (Faverdin, 1985) ou faibles productrices (Bines, Hart et Morant, 1983). La perfusion d'acides gras longs diminue temporairement les quantités ingérées (Vandermeerschen-Doizé et Paquay, 1984).

Enfin, il faut souligner, comme le propose Lindsay (1977), que des variations de paramètres sanguins liées aux repas (baisse de la glycémie (De Jong, 1981c), ou de I'aminoacidémie (Anderson, Mangan et Wright, 1974) peuvent avoir lieu en leur absence, lorsque la distribution de la ration est exceptionnellement différée. Cette accoutumance n'est cependant pas systématique et elle n'a pas été observée dans nos essais sur vaches ; il n'est pas exclu cependant que la prise d'aliments régulière induise des mécanismes réflexes susceptibles d'affecter l'utilisation de certains métabolites même en l'absence de repas.

Les variations des constituants sanguins observées au cours du repas et les faibles effets sur la prise alimentaire obtenus par administration des métabolites sanguins semblent indiquer que le rôle de ces derniers dans le contrôle à court terme de la satiété est limité. Par contre, lorsque la disponibilité de l'un quelconque de ces substrats devient limitante, il est possible que des cellules sensibles au ralentissement métabolique qui s'ensuit provoquent l'initiation d'un repas. Un élément en faveur de cette hypothèse a été fourni par l'étude des variations du métabolisme chez le rat (Nicolaidis et Even, 1984). Les mécanismes provoquant le déclenchement des repas, encore peu étudiés chez les ruminants, mériteraient d'être approfondis.

\section{b) Modifications hormonales.}

- Hormones pancréatiques. - L'insulinémie est beaucoup plus sensible à la prise d'aliments que la glycémie (sur bovins : MacAtée et Trenkle, 1971 ; Vasilatos et Wangsness, 1980 ; Faverdin, 1985 ; sur ovins : Trenkle, 1970 ; Bassett, 1974 ; sur caprins : De Jong, 1981a). L'augmentation de l'insulinémie est précoce : elle précède l'arrivée des nutriments (Bassett, 1974 ; De Jong, 1981a ; Faverdin, 1986a), puis se prolonge en fonction de la durée du grand repas et de la composition du mélange des AGV produit dans le rumen, le propionate étant un facteur beaucoup plus insulinotrope que l'acétate (Horino et al., 1968 ; Ambo, Takahashi et Tsuda, 1973 ; De Jong, 1981c ; Istasse et Orskov, 1984 ; Bines et Hart, 1984). L'insulinémie augmente en fonction de la proportion d'aliments concentrés dans la ration (Lofgreen et Warner, 1972 ; Jenny, Polan et Thye, 1974). Au cours des repas spontanés, l'insulinémie augmente dès les 5 premières minutes du repas (Chase, Wangsness et Martin, 1977 ; De Jong, 1981b), la durée du pic de sécrétion d'insuline, plutôt que son amplitude, étant corrélée avec la durée des petits repas (Vasilatos et Wangsness, 1980).

Chez les ruminants, l'injection intra-veineuse de faibles doses d'insuline provoque un effet de satiété transitoire de 15 à $25 \%$ aussi bien chez le mouton (Deetz et Wangsness, 1981) que chez la vache (Faverdin, 1986b). L'augmentation de la dose injectée stimule la prise alimentaire pendant 2 à 4 h (fig. 6), bien qu'à terme, 
l'encombrement du réticulo-rumen ne permette pas d'induire des hyperphagies durables comme chez le rat (Müller et Collenbrander, 1969 ; Baile et Martin, 1971).

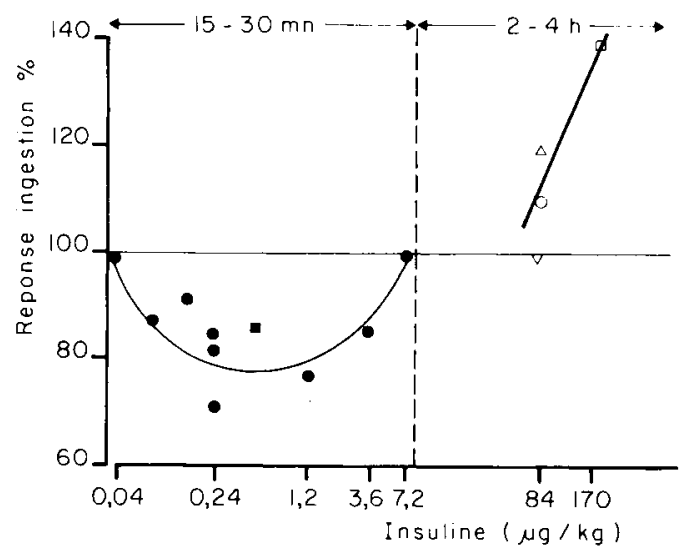

FIG. 6. - Effet de l'injection d'insuline sur la prise alimentaire à court terme en fonction de la dose utilisée (résultats de la bibliographie).

Chez le mouton (Bassett, 1972 ; Ostaszewski et Bares, 1979) et chez la vache laitière (Thilsted, 1985) la prise d'aliments s'accompagne d'une montée de la glucagonémie selon une cinétique très voisine de celle de l'insuline. Cette augmentation n'a pas été observée chez la chèvre, même dans le sang porte (De Jong, 1981a). La sécrétion de glucagon au cours du repas permet de favoriser la néoglucogenèse hépatique à partir du propionate et évite une baisse exagérée de la glycémie par I'insuline (Bassett, 1978). La glucagonémie augmente également au cours des petits repas, mais plus faiblement que I'insulinémie (De Jong, 1981b).

Chez les monogastriques, l'injection de glucagon diminue la prise alimentaire (Langhans et al., 1982) et l'administration d'anticorps a un effet inverse. Chez le mouton, l'ingestion sur $24 \mathrm{~h}$ est réduite (Deetz et Wangsness, 1981) après une injection de glucagon dont le rôle dans le contrôle de la satiété mérite donc d'être approfondi.

- Hormones gastro-intestinales. - La concentration du sang en hormones gastro-intestinales circulantes du groupe gastrine-cholécystokinine (CCK) a été surtout étudiée chez les espèces monogastriques. La prise d'aliment est associée à une augmentation immédiate de la concentration plasmatique de la CCK ainsi que, légèrement différée, de celles du GIP et de l'entéroglucagon (revues de Walsh, 1981 ; Uttenthal, 1985). Les variations de la concentration plasmatique de la gastrine, de la CCK et de la sécrétine associées à la prise d'aliments chez le veau préruminant disparaissent lorsqu'il devient ruminant (Guilloteau et al., 1984, 1985, 1986). Ces variations sont donc à préciser chez le ruminant en liaison avec le repas.

Les différentes formes de CCK, et en particulier l'octapeptide terminal de la CCK (CCK8), apparaissent comme de puissants inhibiteurs de la prise d'aliments à court terme chez le mouton (McLaughlin, 1982 ; Baile, Della-Fera et McLaughlin, 1983). La bombésine, proche du GIP, a également été identifiée comme un inhibi- 
teur de l'ingestion. Pour ces hormones, le problème reste de savoir si les doses efficaces sont proches de leurs variations endogènes au cours du repas.

- Autres modifications hormonales. - La concentration plasmatique d'ailleurs très irrégulière, de l'hormone de croissance (Vasilatos et Wangsness, 1981), est diminuée au cours du grand repas chez le mouton (Bassett, 1974) et la vache laitière (Faverdin, 1985). Cette diminution n'a pas été retrouvée par Bines, Hart et Morant (1983) et Thilsted (1985), bien qu'elle soit présente au cours des petits repas selon De Jong (1981b) et Vasilatos et Wangsness (1980). Les repas spontanés sont fréquemment précédés d'un pic plasmatique d'hormone de croissance dans $70 \%$ des cas chez le mouton (Driver et Forbes, 1978) et $22 \%$ des cas chez la chèvre (De Jong, 1981c). La libération d'hormone de croissance n'influe sans doute pas directement sur la prise d'aliments ; elle pourrait traduire l'apparition de facteurs métaboliques limitants conduisant au déclenchement des repas.

Enfin, les variations des concentrations plasmatiques de prolactine, de thyroxine et de corticostéroïdes (Bines, Hart et Morant, 1983 ; Thilsted, 1985 ; Bassett, 1974) ne sont pas liées de façon étroite à la prise alimentaire.

En conclusion les connaissances concernant les modalités de l'ingestion alimentaire chez les ruminants ont fait des progrès considérables. II reste cependant beaucoup à faire pour les affiner et en présenter des modèles cohérents.

Les ruminants passent beaucoup de temps à mastiquer des aliments qui, en règle générale, sont digérés lentement. Dans ces conditions les phénomènes comportementaux, moteurs et digestifs, jouent un rôle important dans la régulation des quantités d'aliments grossiers ingérées. Les vitesses d'ingestion, de digestion et de transit influencent directement le niveau d'ingestion, dans la mesure où la capacité du rumen est limitée. L'état de réplétion de ce dernier joue donc un rôle fondamental, même si le niveau de réplétion semble relatif et variable au cours de la journée et d'un aliment à l'autre.

Le contrôle de la prise alimentaire, que ce soit au niveau du repas ou de la journée, n'est cependant pas sous la dépendance d'un seul mécanisme. Les métabolites sanguins, qui ont été beaucoup étudiés pour préciser leur rôle dans l'ingestion des aliments riches en énergie, ne constituent pas non plus les facteurs essentiels intervenant dans l'apparition de la satiété chez les ruminants.

Le rôle des phénomènes sensoriels est mal connu dans les situations où l'animal n'a pas le choix entre différents aliments. II mériterait d'être plus étudié, ainsi que celui de produits présents dans les aliments et ayant un effet direct sur l'appétence.

Enfin, la sécrétion des hormones pancréatiques en début de grand repas ou au cours des petits repas, semble être davantage sous la dépendance de stimulations neuro-hormonales associées à la prise d'aliments qu'à l'arrivée des nutriments. Les variations rapides de la sécrétion de ces hormones constituent vraisemblablement des signaux importants pour le contrôle de la prise alimentaire à l'échelle du repas. 


\section{Références}

ALDERSON N. E., MITCHELL G. E., LITTLE J. C. O., CALL J. L., 1972. Post-prandial patterns of ovine ruminoreticular motility and digesta passage. J. anim. Sci., 35, 102-108.

AMBO K., TAKAHASHI H., TSUDA T., 1973. Effects of feeding and infusion of short-chain fatty acids and glucose on plasma insulin and blood glucose levels in sheep. Tohoku J. agric. Res., 24, 54-62.

ANDERSON D. M., MANGAN J. L., WRIGHT P. C., 1974. The relation between insulin and glucose and the concentration of amino acids in plasma and erytrocytes in sheep after feeding. Proc. Nutr. Soc., 33, 79 (Abstr.).

BAILE C. A., 1971. Metabolites as feedbacks for control of feed intake and receptor sites in goats and sheep. Physiol. Behav., 7, 819-826.

BAILE C. A., 1975. Control of feed intake in ruminants, 333-350. In McDONALD I. W., WARNER A. C. I., Digestion and metabolism in the ruminant. The Univ. New Engl. Publ. Unit.

BAILE C. A., 1979. Taste, Appetite and Regulation of Energy balance and control of food intake. CHURCH D. C., Part II. In Digestive physiology and nutrition of ruminants, vol. 2, Nutrition. Sec. Ed. Oxford Press., 291-320.

BAile C. A., Della-ferA M. A., McLAughlin C. L., 1983. Hormones and feed intake. Proc. Nutr. Soc., 42, 113-127.

BAILE C. A., FORBES J. M., 1974. Control of feed intake and regulation of energy balance in ruminants. Physiol. Rev., 54, 161-214.

BAILE C. A., MARTIN F. H., 1971. Hormones and amino acids as possible factors in the control of hunger and satiety in sheep. J. Dairy Sci., 54, 897-905.

BAILE C. A., MAYER J., 1968. Effects of intravenous versus intraruminal injections of acetate on feed intake of goats. J. Dairy Sci., 51, 1490-1494.

BAILEY C. B., 1961. Saliva secretion and its relation to feeding in cattle. 3 - The rate of secretion of mixed saliva in the cow during eating, with an estimate of the magnitude of the total daily secretion of mixed saliva. Brit. J. Nutr., 15, 443-451.

BAILEY C. B., BALCH C. C., 1961a. Saliva secretion and its relation to feeding in cattle. 1 - The composition and rate of secretion of parotid saliva in a small steer. Brit. J. Nutr., 15, 371-382.

BAILEY C. B., BALCH C. C., 1961b. Saliva secretion and its relation to feeding in cattle. 2 - The composition and rate of secretion of mixed saliva in the cow during rest. Brit. J. Nutr., 15 , 383-402.

BALCH C. C., 1958. Observations on the act of eating in cattle. Brit. J. Nutr., 12, 330-345.

BALCH C. C., CAMPLING R. C., 1962. Regulation of voluntary food intake in ruminants. Nutr. Abstr. Rev., 32, 669-686.

BASSETT J. M., 1972. Plasma glucagon concentrations in sheep : their regulation and relation to concentrations of insulin and growth hormone. Aust. J. biol. Sci., 25, 1277-1287.

BASSETT J. M., 1974. Diurnal patterns of plasma insulin, growth hormone, corticosteroid and metabolite concentrations in fed and fasted sheep. Aust. J. biol. Sci., 27, 167-181.

BASSETT J. M., 1978. Endocrine factors in the control of nutrient utilization ruminants. Proc. Nutr. Soc., 37, 273-280.

BINES J. A., DAVEY A. W. F., 1970. Voluntary intake, digestion, rate of passage, amount of material in the alimentary tract and behaviour in cows receiving complete diets containing straw and concentrates in different proportions. Brit. J. Nutr., 24, 1013-1028.

BINES J. A., DAVEY A. W. F., 1978. Metabolic changes associated with intake by cows of complete diets containing straw and concentrates in different proportions. Br. J. Nutr., 39, 567578.

BINES J. A., HART I. C., 1984. The response of plasma insulin and other hormones to intraruminal infusion of VFA mixtures in cattle. Can. J. anim. Sci., 64, 304-305.

BINES J. A., HART I. C., MORANT S. V., 1983. Endocrine control of energy metabolism in the cow : diurnal variations in the concentrations of hormones and metabolites in blood plasma of beef and dairy cows. Horm. Metab. Res., 15, 330-334.

BLAXTER K. L., WAINMAN F. W., WILSON R. S., 1961. The regulation of food intake by sheep. Anim. Prod., 3, 51-61. 
BUÉNO L., RUCKEBUSCH Y., 1974. The cyclic motility of the omasum and its control in sheep. J. Physiol., (London), 238, 295-312.

CAMPLING R. C., 1966. A preliminary study of the effect of pregnancy and of lactation on the voluntary intake of food by cows. Brit. J. Nutr., 20, 25-39.

CAMPLING R. C., BALCH C. C., 1961. Factors affecting the voluntary intake of food by cows. 1 - Preliminary observations on the effect, on the voluntary intake of hay, of changes in the amount of the reticulo-rumen contents. Brit. J. Nutr., 15, 523-530.

CAMPLING R. C., FREER M., 1962. The effect of specific gravity and size on the mean time of retention of inert particles in the alimentary tract of the cow. Brit. J. Nutr., 16, 507-518.

CAMPLING R. C., FREER M., BALCH C. C., 1961. Factors affecting the voluntary intake of food by cows. 2 - The relationship between the voluntary intake of roughages, the amount of digesta in the reticulo-rumen and the rate of disappearance of digesta from the alimentary tract. Brit. J. Nutr., 15, 531-540.

CAMPLING R. C., FREER M., BALCH C. C., 1962. Factors affecting the voluntary intake of food by cows. 3 - The effect of urea on the voluntary intake of oat straw. Brit. J. Nutr., 16, 115-124.

CAMPLING R. C., FREER M., BALCH C. C., 1963. Factors affecting the voluntary intake of food by cows. 6 - A preliminary experiment with ground, pelleted hay. Brit. J. Nutr., 17, 263-272.

CARLE B., DULPHY J. P., 1980. Comportement alimentaire comparé des ovins et des bovins. Relation avec la digestion des aliments. Reprod. Nutr. Dévelop., 20, 1633-1639.

CHASE L. E., WANGSNESS P. J., BAUMGARDT B. R., 1976. Feeding behavior of steers fed a complete mixed ration. J. Dairy Sci., 59, 1923-1928.

CHASE L. E., WANGSNESS P. J., MARTIN R. J., 1977. Portal blood insulin and metabolite changes with spontaneous feeding in steers. J. Dairy Sci, 60, 410-415.

CIRIO MAISONNAVE A., BOIVIN R., BOST J., 1981. Stimulation prandiale de la motricité réticulaire chez le mouton : phase céphalique et réflexe oral. Ann. Rech. vét., 12, 291-302.

CONRAD H. R., PRATT A. D., HIBBS J. W., 1964. Regulation of feed intake in dairy cows. I Change in importance of physical and physiological factors with increasing digestibility. $J$. Dairy Sci., 47, 54-67.

DE JONG A., 1981a. The effect of feed intake on nutrient and hormone levels in jugular and portal blood in goats. J. agric. Sci, 96, 643-657.

DE JONG A., 1981b. Short- and long-term effects of eating on blood composition in free-feeding goats. J. agric. Sci., 96, 659-668.

DE JONG A., 1981c. Regulation of food intake in the goat: circulating metabolites and hormones in relation to eating. Thèse Doct., Univ. Groningen, $151 \mathrm{p}$.

DECAEN C., JOURNET M., 1967. Evolution au début de la lactation de la sécrétion des principaux acides gras du lait et de la concentration en acides gras libres du sang chez la vache. Ann. Biol. anim. Bioch. Biophys., 7, 131-143.

DEETZ L. E., WANGSNESS P. J., 1981. Influence of intrajugular administration of insulin, glucagon and propionate on voluntary feed intake of sheep. J. anim. Sci., 53, 427-433.

DESWYSEN A., 1980. Influence de la longueur des brins et de la concentration en acides organiques des ensilages sur l'ingestion volontaire chez les ovins et les bovins. Thèse Doct. Université Catholique de Louvain. Belgique. $254 \mathrm{p}$.

DESWYSEN A. G., EHRLEIN H. J., 1981. Silage intake, rumination and pseudo-rumination activity in sheep studied by radiography and jaw movement recordings. Brit. J. Nutr., 46, 327-335.

DOREAU M., REMONO B., 1982. Comportement alimentaire et utilisation disgestive d'une ration de composition constante chez la vache laitière en fin de gestation et début de lactation. Reprod. Nutr. Dévelop., 22, 307-324.

DRIVER P. M., FORBES J. M., 1978. Plasma growth hormone and spontaneous meal in sheep. Proc. Nutr. Soc., 37, 100A.

DULPHY J. P., 1985. Etude des quantités ingérées lors des grands repas chez des moutons recevant des fourrages ensilés. Ann. Zootech., 34, 401-416.

DULPHY J. P., BECCHET G., 1976. Influence du stade de végétation et de l'espèce végétale sur le comportement alimentaire et mérycique de moutons recevant des fourrages verts hachés. Ann. Zootech., 25, 505-519.

DULPHY J. P., BÉCHET G., THOMSON E., 1975. Influence de la structure physique et de la qualité de conservation des ensilages de graminées sur leur ingestibilité. Ann. Zootech., 24, 81-94. 
DULPHY J. P., CARLE B., 1986. Activités alimentaires et méryciques comparées des bovins, des caprins et des ovins. Reprod. Nutr. Dévelop., 26, 279-280.

DULPHY J. P., MICHALET-DOREAU B., 1983. Comportement alimentaire et mérycique d'ovins et de bovins recevant des fourrages verts. Ann. Zootech., 32, 465-474.

DULPHY J. P., MICHALET-DOREAU B., DEMARQUILLY C., 1984. Etude comparée des quantités ingérées et du comportement alimentaire et mérycique d'ovins et de bovins recevant des ensilages d'herbe réalisés selon différentes techniques. Ann. Zootech., 33, 291-320.

DULPHY J. P., RÉMOND B., THÉRIEZ M., 1979. Ingestive behaviour and related activities in ruminants, 103-122. In RUCKEBUSCH Y., THIVEND P. Digestion and metabolism in ruminants. MTP Ltd Press. Lancaster. England.

EGAN A. R., 1970. Nutritional status and intake regulation in sheep. VI - Evidence for variation in setting of an intake regulatory mechanism relating to the digesta content of the reticulorumen. Aust. J. agric. Res., 21, 735-746.

FAVERDIN P., 1985. Régulation de l'ingestion des vaches laitières en début de lactation : variations au cours du nycthémère de l'activité alimentaire, des métabolites sanguins et de l'insulinémie - étude du rôle de l'insuline. Thèse de Doct. Ingén., I.N.A.P.G., 131 p.

FAVERDIN P., 1986a. Variations de l'insulinémie en début de repas chez la vache en lactation. Reprod. Nutr. Dévelop., 26, 381-382.

FAVERDIN P., 1986b. Injections de doses physiologiques d'insuline chez la vache en lactation : effets sur les quantités ingérées et les métabolites sanguins. Reprod. Nutr. Dévelop., 26, 383384.

FOCANT M., GALLOUIN F., Is/8. Effet de la perfusion des acides gras volatils sur la prise alimentaire et la rumination chez la chèvre. $J$. Physiol. Paris, 74, 19A.

FORBES J. M., 1977a. Inter-relationships between physical and metabolic control of voluntary food intake in fattening, pregnant and lactating mature sheep : a model. Anim. Prod., 24, 91-101.

FORBES J. M., 1977b. Development of a model of voluntary food intake and energy balance in lactating cows. Anim. Prod., 24, 203-214.

FORBES J. M., 1980. A model of the short-term control of feeding in the ruminant : effects of changing animal or feed characteristics. Appetite, 1, 21-41.

FORBES J. M., 1980. Hormones and metabolites in the control of food intake, 145-160. In RUCKEBUSCH Y., THIVEND P., Digestive physiology and metabolism in the ruminant, Symp. ClermontFerrand (France), MTP Ltd Press, Lancaster.

FREER M., CAMPLING R. C., 1963. Factors affecting the voluntary intake of food by cows. 5 - The relationship between the voluntary intake of food, the amount of digesta in the reticulo-rumen and the rate of disappearance of digesta from the alimentary tract with diets of hay, dried grass or concentrates. Brit. J. Nutr., 17, 79-88.

FREER M., CAMPLING R. C., 1965. Factors affecting the voluntary intake of food by cows. $7-$ The behavior and reticular motility of cows given diets of hay, dried grass, concentrates and ground, pelleted hay. Brit. J. Nutr., 19, 195-207

FREER M., CAMPLING R. C., BALCH C. C., 1962. Factors affecting the voluntary intake of food by cows. 4 - The behaviour and reticular motility of cows receiving diets of hay, oat straw and oat straw with urea. Brit. J. Nutr., 16, 279-295.

GALLOUIN F., FOCANT M., 1980. Bases physiologiques du comportement alimentaire chez les ruminants. Reprod. Nutr. Dévelop., 20, 1563-1614.

GATEL F., 1984. Signification de la satiété à court terme chez le mouton : influence de la qualité du fourrage et des stimuli associés à la prise de nourriture. Ann. Zootech., 33, 111-118.

GILL J., CAMPLING R. C., WESTGARTH D. R., 1966. A study of chewing during eating in the cow. Brit. J. Nutr., 20, 13-23.

GORDON J. G., 1968. Rumination and its significance. World Rev. Nut. Diet., 9, 251-273.

GORDON J. G., Mc ALLISTER I. K., 1970. The circadian rythm of rumination. J. agric. Sci. Camb., 74, 291-297.

GREENHALGH J. F. D., REID G. W., 1971. Relative palatibility to sheep of straw, hay and dried grass. Brit. J. Nutr., 26, 107-116

GRENET E., 1987. Etude comparée de la vitesse de vidange du rumen chez le mouton alimenté avec des foins de graminées et de légumineuses. Reprod. Nutr. Dévelop., 27, 229-230. 
GROVUM W. L., 1979. Factors affecting the voluntary intake of food by sheep. 2 - The role of distension and tactile input from compartments of the stomach. Brit. J. Nutr., 42, 425-436.

GUÉRIN H., DULPHY J. P., 1984. Influence de l'apport complémentaire de maïs, de pulpe de betterave ou de mélasse sur la valeur alimentaire d'un foin. Ann. Zootech., 33, 509-532.

GUILLOTEAU P., CHAYVIALLE J. A., TOULLEC R., GRONGNET J. F., DARDILLAT C., 1985. Evolution du taux circulant de gastrine chez le veau. Reprod. Nutr. Dévelop., 25, 780.

GUILLOTEAU P., CHAYVIALLE J. A., TOULLEC R., GRONGNET J. F., DARDILLAT C., BERNARD C., 1986. Evolution du taux plasmatique de CCK avec l'âge et le régime alimentaire chez le jeune veau. Reprod. Nutr. Dévelop., 26, 377.

GUILLOTEAU P., CHAYVIALLE J. A., TOULLEC R., GRONGNET J. F., DARDILLAT C., 1984. Earlylife pattern of plasma secretin level in calves. Can. J. Anim. Sci., 64 (Suppl.), 100-101.

HIDARI H., 1979. Estimation of the rumen load of sheep through measuring the consistency of rumen contents. Jap. J. zootech. Sci., 50, 402-410.

HIDARI H., 1984. Effect of altering the rumen load on the feeding behavior in sheep. Can. $J$. anim. Sci., 64 (Suppl.), 320-321.

HOLMES C. W., MCLEAN N. A., LOCKYER K. J., 1978. Changes in the rate of heat production of calves during grazing and eating. N.Z. J. agric. Res., 21, 107-112.

HORINO M., MACHLIN L. J., HERTELENDY F., KIPNIS D. M., 1968. Effect of short-chain fatty acids on plasma insulin in ruminant and non-ruminant species. Endocrinology, 83, 118-128.

HOUPT T. R., 1974. Stimulation of food intake in ruminants by 2-deoxy-D-glucose and insulin. Am. J. Physiol., 227, 161-167.

HUNTINGTON G. B., 1982. Portal blood flow and net absorption of ammonia nitrogen, urea nitrogen and glucose in non-lactating Holstein cows. J. Dairy Sci, 65, 1155-1162.

IGGO A., 1960. Cutaneous mechanoreceptors with afferent $C$ fibres. J. Physiol., 152, 337-353.

I.N.R.A., 1978. Alimentation des Ruminants. Ed. I.N.R.A. Publications (Route de Saint-Cyr) 78000 Versailles, France, $597 \mathrm{p}$.

ISTASSE L., ØRSKOV E. R., 1984. The effects of intermittent and continous infusions of propionic acid on plasma insulin. Can. J. anim. Sci, 64 (suppl.), 148-149.

JASTER E. H., MURPHY M. R., 1983. Effects of varying particle size of forage on digestion and chewing behavior of dairy heifers. J. Dairy Sci., 66, 802-810.

JENNY B. F., POLAN C. E., THYE F. W., 1974. Effects of high grain feeding and stage of lactation on serum insulin, glucose and milk fat percentage in lactating cows. J. Nutr., 104, 379-385.

JONES G. M., 1972. Chemical factors and their regulation to feed intake regulation in ruminants: a review. Can. J. anim. Sci, 52, 207-239.

JOURNET M.. JARRIGE R., 1970. Facteurs physiologiques de la quantité d'aliment ingérée par les Ruminants. XXVle Journ. de la Féd. Europ. de Zootechnie, Budapest.

JOURNET M., RÉMOND B., 1976. Physiological factors affecting the voluntary intake of feed by cows : a review. Livest. Prod. Sci., 3, 129-146.

KAUFMANN W., ORTH A., 1966. Untersuchungen über Einflüsse des Futters und der Pansenfermentation auf die Spiechelsekretion. Z. Tierphysiol. Tierernährg. u. Futtermittelkde, 21, 110120.

KENNEDY P. M., 1985. Effect of rumination on reduction of particle size of rumen digesta by cattle. Aust. J. agric. Res., 36, 819-828.

KENNEY P. A., BLACK J. L., 1984. Factors affecting diet selection by sheep. IV - Level of feeding. Aust. J. agric. Res., 35, 839-843.

LANGHANS W., ZIEGER V., SCHARRER E., GEARY N., 1982. Stimulation of feeding in rats by intraperitoneal injection of antibodies to glucagon. Science, 218, 894-895.

LAREDO M. A., MINSON D. J., 1973. The voluntary intake, digestibility, and retention time by sheep of leaf and stem fractions of five grasses. Aust. J. agric. Res., 24, 875-888.

LAWLOR M. J., GIESECKE D., WALSER-KÄRST K., 1966. Comparative studies on the digestive physiology of sheep fed on semi-purified or roughage-concentrate diets. 1 - Food and water intake, rumen volume and rates of parotid secretion. Brit. J. Nutr., 20, 373-382.

LEEK B. F., 1969. Reticulo-ruminal mechanoreceptors in sheep. J. Physiol. (London), 202, 585-609. LE MAGNEN J., 1970. Insuline et prise alimentaire. In KLOTZ H. P., Les hormones et le comportement. L'Expansion. 
LINDSAY D. B., 1977. The effect of feeding pattern and sampling procedure on blood parameters. In LISTER D., Blood profiles in animal production, Occasio., Publ. No 1 Brit. Soc. Anim. Prod., p. 99-120.

LOFGREEN P. A., WARNER R. G., 1972. Relationship of dietary caloric density and certain blood metabolites to voluntary feed intake in mature wethers. J. anim. Sci., 356, 1239-1247.

LOMAX M. A., BAIRD G. D., 1983. Blood flow and nutrient exchange across liver and gut of the dairy cow. Effect of lactation and fasting. Br. J. Nutr., 49, 481-496.

MCATEE I. W., TRENKLE A., 1971. Metabolic regulation of plasma insulin levels in cattle. $J$. anim. Sci., 33, 438-442.

MCLAUGHLIN C. L., 1982. Role of peptides from gastrointestinal cells in food intake regulation. $J$. anim. Sci., 55, 1515-1527.

METZ J. H. M., 1975. Time patterns of feeding and rumination in domestic cattle. Meded. Landbouwhogesch. Wageningen, 75-12.

MONTGOMERY M. J., BAUMGARDT B. R., 1965. Regulation of food intake in ruminants. 1 - Pelleted rations varying in energy concentration. J. Dairy Sci., 48, 569-574.

MÜLLER L. D., COLENBRANDER V. F., 1969. Effect of insulin administration on feed intake of sheep. J. Dairy Sci., 52, 907-908.

MURPHY M. R., BALDWIN R. L., ULYATT M. J., KOONG L. J., 1983. A quantitative analysis of rumination patterns. $J$. anim. Sci., 56, 1236-1240.

NICOLAIDIS S., EVEN P., 1984. Mesure du métabolisme de fond en relation avec la prise alimentaire : hypothèse ischymétrique. C. R. Acad. Sci. Paris, 298, 295-300.

ORTH A., KAUFMANN W., 1966. Zur Wirkung von Bicarbonat auf die Futteraufnahme bei Milchkühen. Z. Tierphysiol. Tiernernährg. u. Futtermittelkde, 21, 350-361.

OSTASZEWSKI P., BARES W., 1979. Influence of rumen fermentation rate on glucagon and insulin blood level. Ann. Rech. vét., 10, 385-387.

OSUJI P. O., GORDON J. G., WEBSTER A. J. F., 1975. Energy exchanges associated with eating and rumination in sheep given grass diets of different physical forms. Br. J. Nutr., 34, 59-71.

PAQUAY R., DE BAERE R., LOUSSE A., 1971. Des variations du poids vif chez la bête bovine. Rev. Agric, 4, 419-429.

PEARCE G. R., MOIR R. J., 1964. Rumination in sheep. I - The influence of rumination and grinding upon the passage and digestion of food. Aust. J. agric. Res., 15, 635-644.

PETIT M., 1972. Emploi du temps des troupeaux de vaches mères et leurs veaux sur les pâturages d'altitude de l'Aubrac. Ann. Zootech., 21, 5-27.

PITON J., 1975. Intérêt et importance de la mobilisation des réserves corporelles des vaches laitières au début de la lactation. Thèse Doct. Montpellier, $51 \mathrm{p}$.

POND K. R., GOODE L., LEONARD E. S., MANN D. L., 1984. Intake, digesta fill and flow kinetics pre and postpartum. Can J. anim. Sci., 64 (suppl.), 68-69.

REID C. S. W., 1963. Diet and the motility of the forestomachs of the sheep. Proc. N. Z. Soc. anim. Prod., 23, 169-188.

RUCKEBUSCH Y., 1963. Recherches sur la régulation centrale du comportement alimentaire chez les ruminants. Thèse Doct. Sci. Univ. Lyon, $213 \mathrm{p}$.

RUCKEBUSCH Y., BUÉNO L., 1972. Analyse quantitative des relations activité alimentaire état de vigilance chez les bovins en stabulation. Ann. Rech. vét., 3, 399-420.

RUCKEBUSCH Y., BUENO L., 1978. An analysis of ingestive behaviour and activity of cattle under field conditions. Appl. anim. Ethol., 4, 301-313.

RUCKEBUSCH Y., BUÉNO L., FIORAMONTI J., 1981. La mécanique digestive chez les mammifères. Actual. Sci. Agron. I.N.R.A./Masson, $131 \mathrm{p}$.

RUCKEBUSCH Y., LAPLACE J. P., 1967. Sur le déterminisme de la rumination. Bull. Soc. Sci. vét. Méd. comp. Lyon, 69, 185-195.

SCOTT D., 1975. Changes in mineral, water and acid-base balance associated with feeding and diet, 205-215. In Digestive physiology and metabolism in ruminants. I.W. Mc DONALD, A.C.I. WARNER, Univ. New Engl. Publ. Unit.

SEOANE J. R., BAILE C. A., MARTIN F. H., 1972. Humoral factors modifying feeding behaviour of sheep. Physiol. Behav., 8, 993-995.

SIMKINS K. L., SUTTIE J. W., BAUMGARDT B. R., 1965. Regulation of food intake in ruminants : 
3. Variation in blood and rumen metabolites in relation to food intake. J. Dairy Sci., 48, 16291634.

SMITH E. G., WEEKS R. W., JARRARD J. W., 1969. Radiotelemetry studies of reticular pressures in free-feeding cattle, 66-75. In A.T. PHILLIPSON, Physiology of digestion and metabolism in the ruminant. Oriel Press, Newcastle upon Tyne.

STEVENS C. E., 1970. Fatty acid transport through the rumen epithelium, 101-112. In Physiology and digestion of the ruminant. Oriel Press Ltd, Newcastle-upon-Tyne, England.

SUZUKI S., HIDARI H., 1973. Effects of the time feed given on the diurnal feeding pattern of dairy cows. Jap. J. zootech. Sci, 44, 216-221.

SUZUKI S., SHINDE Y., HIDARI H., 1970. Effects of a change in the daily time of access to hay on the rate of eating and feed intake of dairy cows. Jap. J. zootech. Sci., 41, 423-429.

SUZUKI S., SHINDE Y., HIDARI H., 1973. Effects of divided feeding of roughage on the rate of eating during a meal in dairy cows. Jap. J. zootech. Sci., 44, 181-187.

TERNOUTH J. H., BEATTIE A. W., 1971. Studies of the food intake of sheep at a single meal. Brit. J. Nutr., 25, 153-164.

THILSTED S. H., 1985. Regulation of the partition of nutrients in the dairy cow in late pregnancy and early lactation. Z. Tierphysiol. Tierernährg $u$. Futtermittelkde, 53, 10-18.

THOMSON B. C., CRUICKSHANK G. J., POPPI D. P., SYKES A. R., 1985. Diurnal patterns of rumen fill in grazing sheep. Proc. N.Z. Soc. anim. Prod,, 45, 117-120.

THORNTON R. F., MINSON D. J., 1972. The relationship between voluntary intake and mean apparent retention time in the rumen. Aust. $J$. agric. Res., 23, 871-877.

THORNTON R. F., MINSON D. J., 1973. The relationship between apparent retention time in the rumen, voluntary intake, and apparent digestibility of legume and grass diets in sheep. Aust. J. agric. Res., 24, 889-898.

TRENKLE A., 1970. Effects of short chain fatty acids, feeding fasting and type of diet on plasma insulin levels in sheep. J. Nutr., 100, 1323-1330.

TULLOH N. M., 1966. Physical studies of the alimentary tract of grazing cattle. IV - Dimensions of the tract in lactating and non lactating cows. N.Z. J. agric. Res., 9, 999-1008.

TULLOH N. M., HUGUES J. W., 1965. Physical studies of the alimentary tract of grazing cattle. II - Techniques for estimating the capacity of the reticulo-rumen. N.Z. J. agric. Res., 8 , 1070-1078.

TULLOH N. M., HUGUES J. W., NEWTH R. P., 1965. Physical studies of the alimentary tract of grazing cattle. 1 - Measurement of volume of water in the reticulo-rumen. N.Z. J. agric. Res., 8, 636-651.

ULYAT M. J., DELLOW D. W., JOHN A., REID C. S. W., WAGHORN G. C., 1986. Contribution of chewing during eating rumination to the clearance of digesta from rumino reticulum, 498515. In Control of digestion and metabolism in ruminants. L.P. MILLIGAN, W. L. GROVUM, A. DOBSON (Eds). Prentice Hall. Englewood Cliffs N.J.

UTTENTHAL L. O., 1985. the gut hormone response to food. Proc. Nutr. Soc., 44, 53-61.

VANDERMEERSCHEN-DOIZÉ F., PAQUAY R., 1984. Etude du contrôle à long terme des ingestions volontaires chez le ruminant. IRSIA, Presses univ. de Namur., $200 \mathrm{p}$.

VASILATOS R., WANGSNESS P. J., 1980. Changes in concentrations of insulin, growth hormone and metabolites in plasma with spontaneous feeding in lactating dairy cows. J. Nutr., 110, 1479-1487.

VASILATOS R., WANGSNESS P. J., 1981. Diurnal variations in plasma insulin and growth hormone associated with two stages of lactation in high producing dairy cows. Endocrinology, 108, 300-304.

VÉRITÉ R., JOURNET M., 1970. Influence de la teneur en eau et de la déshydratation de l'herbe sur sa valeur alimentaire par les vaches laitières. Ann. Zootech., 19, 255-268.

WAGHORN G. C., REID C. S. W., 1977. Rumen motility in sheep and cattle as affected by feeds and feeding. Proc. N.Z. Soc. anim. Prod., 37, 176-181.

WALSH J. H., 1981. Gastrointestinal hormones and peptides. In : L.R. JOHNSON. Physiology of the gastro-intestinal tract. Vol. 1, p. 59-144. Raven Press, New-York.

WANGSNESS P. J., CHASE L. E., PETERSON A. D., HARTSOCK T. G., KELLMEL D. J., BAUMGARDT B. R., 1976. System for monitoring feeding behavior of sheep. J. anim. Sci., 42, 1544-1549. 
WEBSTER A. J. F., 1974. Clearance by the hepatic portal circulation of the products of digestion and metabolism in the ruminant gut. Proc. Nutr. Soc., 33, 155-161.

WEBSTER A. J. F., 1980. Energy costs of digestion and metabolism in the gut, 145-160. In RUCKEBUSCH Y., THIVEND P., Digestive physiology and metabolism in the ruminant, Symp. Clermont-Ferrand (France), MTP Ltd Press, Lancaster, England.

WEEKES T. E. C., 1971. Role of the rumen mucosa in the sheep : relative changes in weight, and in the ability to metabolize propionate, during pregnancy and lactation. Res. vet. Sci., 12, 373376.

WEEKES T. E. C., WEBSTER A. J. F., 1975. Metabolism of propionate in tissues of sheep gut. Br. J. Nutr., 33, 425-438.

WELCH J. G., 1982. Rumination, particle size and passage from the rumen. J. anim. Sci., 54, 885894.

WELCH J. G., SMITH A. M., 1969. Effect of varying amounts of forage intake on rumination. $J$. anim. Sci., 28, 827-830.

WILSON A. D., 1963. The effect of diet on the secretion of parotid saliva by sheep. II - Variations in the rate of salivary secretion. Aust. J. agric. Res., 14, 680-689.

WILSON A. D., TRIBE D. E., 1963. The effect of diet on the secretion of parotid saliva by sheep. 1 - The daily secretion of saliva by caged sheep. Aust. J. agric. Res., 14, 670-679.

WYBURN R. S., 1979. The mixing and propulsion of the stomach contents of ruminants, 35-51. In Digestive physiology and metabolism of ruminants. Y. RUCKEBUSCH, P. THIVEND (Ed.) MTP Ltd Press Lancaster, England.

XANDÉ A., 1978. Valeur alimentaire des pailles de céréales chez le mouton : influence de la complémentation azotée et énergétique sur l'ingestion et l'utilisation digestive d'une paille d'orge. Ann. Zootech., 27, 583-599. 